\title{
GENERATION AND PROPAGATION OF INTERFACES IN REACTION-DIFFUSION SYSTEMS
}

\author{
XINFU CHEN
}

ABSTRACT. This paper is concerned with the asymptotic behavior, as $\varepsilon \searrow 0$, of the solution $\left(u^{\varepsilon}, v^{\varepsilon}\right)$ of the second initial-boundary value problem of the reaction-diffusion system:

$$
\left\{\begin{array}{l}
u_{t}^{\varepsilon}-\varepsilon \Delta u^{\varepsilon}=\frac{1}{\varepsilon} f\left(u^{\varepsilon}, v^{\varepsilon}\right) \equiv \frac{1}{\varepsilon}\left[u^{\varepsilon}\left(1-u^{\varepsilon 2}\right)-v^{\varepsilon}\right], \\
v_{t}^{\varepsilon}-\Delta v^{\varepsilon}=u^{\varepsilon}-\gamma v^{\varepsilon}
\end{array}\right.
$$

where $\gamma>0$ is a constant. When $v \in(-2 \sqrt{3} / 9,2 \sqrt{3} / 9), f$ is bistable in the sense that the ordinary differential equation $u_{t}=f(u, v)$ has two stable solutions $u=h_{-}(v)$ and $u=h_{+}(v)$ and one unstable solution $u=h_{0}(v)$, where $h_{-}(v), h_{0}(v)$, and $h_{+}(v)$ are the three solutions of the algebraic equation $f(u, v)=0$. We show that, when the initial data of $v$ is in the interval $(-2 \sqrt{3} / 9,2 \sqrt{3} / 9)$, the solution $\left(u^{\varepsilon}, v^{\varepsilon}\right)$ of the system tends to a limit $(u, v)$ which is a solution of a free boundary problem, as long as the free boundary problem has a unique classical solution. The function $u$ is a "phase" function in the sense that it coincides with $h_{+}(v)$ in one region $\Omega_{+}$and with $h_{-}(v)$ in another region $\Omega_{-}$. The common boundary (free boundary or interface) of the two regions $\Omega_{-}$and $\Omega_{+}$moves with a normal velocity equal to $\mathscr{V}(v)$, where $\mathscr{V}(\cdot)$ is a function that can be calculated. The local (in time) existence of a unique classical solution to the free boundary problem is also established. Further we show that if initially $u(\cdot, 0)-h_{0}(v(\cdot, 0))$ takes both positive and negative values, then an interface will develop in a short time $O(\varepsilon|\ln \varepsilon|)$ near the hypersurface where $u(x, 0)-h_{0}(v(x, 0))=0$.

\section{INTRODUCTION}

This paper is concerned with the interfacial phenomena in the reactiondiffusion system

$$
\begin{aligned}
& u_{t}=\varepsilon \Delta u+\frac{1}{\varepsilon} f(u, v) \\
& v_{t}=\Delta v+g(u, v)
\end{aligned}
$$

Received by the editors September 28, 1990.

1991 Mathematics Subject Classification. Primary 35B25; Secondary 35B40, 35R35, 35K45, $35 \mathrm{~K} 50$.

Key words and phrases. Reaction-diffusion systems, generation of interface, propagation of interface.

This work was completed when the author was a graduate student at the University of Minnesota. The author thanks Professor Avner Friedman for his direction of this work and the Alfred P. Sloan Doctoral Dissertation Fellowship for its financial support during the 1990-1991 academic year. 
with

$$
\begin{gathered}
f(u, v)=F(u)-v, \quad F(u)=u\left(1-u^{2}\right), \\
g(u, v)=u-\gamma v
\end{gathered}
$$

where $\gamma>0$ is a constant and $\varepsilon>0$ serves as a small parameter.

The system (1.1), (1.2) models the propagation of chemical waves in excitable media where $u$ is a propagator and $v$ is a controller (see Fife and Tyson [21] for a physical description of this system; Ohta, Mimura, and Kobayashi [28] also used this system to describe an activator-inhibitor model).

The assumption that $\varepsilon$ is small means that the propagator $u$ diffuses quite slowly while its reaction takes place much faster. When $v \in(-2 \sqrt{3} / 9,2 \sqrt{3} / 9)$, the algebraic equation $f(u, v)=0$ has three solutions

$$
u=h_{-}(v), \quad u=h_{0}(v), \quad \text { and } \quad u=h_{+}(v)
$$

where $h_{-}(v)<h_{0}(v)<h_{+}(v)$. In this paper, we are only interested in the case when $v \in(-2 \sqrt{3} / 9,2 \sqrt{3} / 9)$. In this case, $f$ is bistable in the sense that the ordinary differential equation $u_{t}=f(u, v)$ has two stable solutions $u=h_{-}(v)$ and $u=h_{+}(v)$ and one unstable solution $u=h_{0}(v)$. The bistable property of $f$ and the smallness of $\varepsilon$ are essential to the so-called interfacial phenomenon described below.

Starting with smooth initial data, the diffusion term $\varepsilon \Delta u$ in (1.1) and the variation of $v$ from its initial data can be neglected for a short time, so that equation (1.1) can be approximated by the ordinary differential equation $u_{t}=$ $\frac{1}{\varepsilon} f(u, v(x, 0))$, and therefore $u(x, t)$ tends quickly to either $h_{+}(v(x, 0))$ or $h_{-}(v(x, 0))$ according to the sign of $u(x, 0)-h_{0}(v(x, 0))$. Thus, after a short time, the space is partitioned into three regions: a region $\Omega_{+}$where $u$ is almost equal to $h_{+}(v)$, a region $\Omega_{-}$where $u$ is almost equal to $h_{-}(v)$, and a "thin" strip region $\Omega_{0}$ which links $\Omega_{-}$and $\Omega_{+}$. The region $\Omega_{0}$ is so thin that it can be considered as a hypersurface, called interface. We refer to the above process as the generation of the interface. Subsequently, if $x$ is away from the interface, the diffusion term $\varepsilon \Delta u$ can still be neglected, and therefore $u(x, t)$ approximately equals $h_{-}(v(x, t))$ or $h_{+}(v(x, t))$ depending on which region $x$ belongs to, whereas $v$ approximately solves (1.2) with $g=g\left(h_{-}(v), v\right)$ in one region and $g=g\left(h_{+}(v), v\right)$ in the other region. On the other hand, near the interface, the change in $u$ is large, so that $\varepsilon \Delta u$ is nonnegligible. In fact, $\varepsilon \Delta u$ will approximately balance the reaction term $-\frac{1}{\varepsilon} f$, and their difference is a force which will drive the interface to move; this motion is called the propagation of the interface. The normal velocity of the motion of the interface will be determined by the speed of a planar travelling wave solution of equation (1.1).

It is well known that (1.1) has a planar travelling wave solution

$$
u=U\left(\frac{x \cdot s-c t}{\varepsilon}, v\right), \quad x \in \mathscr{R}^{n}, s \in S^{n-1} \subset \mathscr{R}^{n}, t \in \mathscr{R},
$$

where $U=U(z, v)$ and $c=\mathscr{V}(v)$ form the unique solution of the nonlinear 
eigenvalue problem:

$$
\left\{\begin{array}{l}
U_{z z}(z, v)+c U_{z}(z, v)+f(U(z, v), v)=0 \quad \forall z \in \mathscr{R}^{1}, \\
\lim _{z \rightarrow+\infty} U(z, v)=h_{+}(v), \\
\lim _{z \rightarrow-\infty} U(z, v)=h_{-}(v), \\
U(0, v)=h_{0}(v) .
\end{array}\right.
$$

(For more detailed discussions of the above eigenvalue problem, see Aronson and Weinberger $[2,3]$.

Fife and Hsiao [19] considered the one-dimensional Cauchy problem for equation (1.1) where $v$ is a known function of $x$. They proved that starting with smooth initial data $u(x, 0)$ satisfying $u(x, 0)>h_{0}(v(x))$ when $x>0$, and $u(x, 0)<h_{0}(v(x))$ when $x<0$, the solution of (1.1) approximates the function $U((x-\xi(t)) / \varepsilon, v(\xi(t))$ as $\varepsilon \rightarrow 0$, where $U(z, v)$ is the solution of (1.5) and $x=\xi(t)$ is a function determined by the motion law

$$
\left\{\begin{array}{l}
\dot{\xi}(t)=\mathscr{V}(v(\xi(t))), \quad t>0, \\
\xi(0)=0 .
\end{array}\right.
$$

When $v=0$, equation (1.1) is known as the Allen-Cahn equation [1]. Its onedimensional case has been extensively studied by Bronsard-Kohn [6], Carr-Pego [7, 8], Fusco [23], Fusco-Hale [24], and the references therein. Recently, some results for the Allen-Cahn equation have been extended to higher dimensions. Here the mean curvature $K$ of the interface takes a role in the propagation of the interface. Formal derivation shows that the normal velocity of the interface is $\varepsilon K$ (see, for example, Allen-Cahn [1], Rubinstein-Sternberg-Keller [30], and Fife [18]). Rigorous proofs were recently given by Bronsard-Kohn [5], DeMottoni-Schatzman [14, 15], Evans-Soner-Souganidis [16], and the author [9]. The method in [9] is based on the construction of comparison functions and is flexible enough to be extended to the system (1.1), (1.2), as we shall do in this paper.

For the system (1.1), (1.2), X.-Y. Chen [10] has recently proven the generation of the interface. Concerning the propagation of the interface, he derived an $\varepsilon$ dependent free boundary problem, proved its local (in time) existence (for any fixed $\varepsilon>0$ ), and then formally showed that the solution of the free boundary problem approximates the solution of (1.1), (1.2). The free boundary problem is to find a function $\tilde{v}^{\varepsilon}$ and a free boundary (interface) $\Gamma^{\varepsilon}$ which separates $\mathscr{R}^{n}$ into two disjoint regions $\Omega_{-}^{\varepsilon}(t)$ and $\Omega_{+}^{\varepsilon}(t)$ at each time $t>0$, such that (1.7)

$$
\left\{\begin{array}{l}
\tilde{v}_{t}^{\varepsilon}-\Delta \tilde{v}^{\varepsilon}=g\left(h_{+}\left(\tilde{v}^{\varepsilon}\right), \tilde{v}^{\varepsilon}\right) \chi_{\Omega_{+}^{\varepsilon}}+g\left(h_{-}\left(\tilde{v}^{\varepsilon}\right), \tilde{v}^{\varepsilon}\right) \chi_{\Omega_{-}^{\varepsilon}}, \quad x \in \mathscr{R}^{n}, t>0, \\
V_{\Gamma^{\varepsilon}}=\mathscr{V}\left(\tilde{v}^{\varepsilon}\right)+\varepsilon K^{\varepsilon}, \\
\tilde{v}^{\varepsilon}(x, 0)=\psi(x), \quad x \in \mathscr{R}^{n}, \\
\Gamma^{\varepsilon}(0)=\left\{x \in \mathscr{R}^{n} \mid \varphi(x)=h_{0}(\psi(x))\right\}, \quad x \in \mathscr{R}^{n},
\end{array}\right.
$$

where $\chi_{A}$ stands for the characteristic function of the set $A$, and $V_{\Gamma^{\varepsilon}}$ and $K^{\varepsilon}$ are respectively the normal velocity and the mean curvature of the free boundary $\Gamma^{\varepsilon}$; the function $\tilde{u}^{\varepsilon}$ is defined by $\tilde{u}^{\varepsilon}=h_{+}\left(\tilde{v}^{\varepsilon}\right) \chi_{\Omega_{+}^{\varepsilon}}+h_{-}\left(\tilde{v}^{\varepsilon}\right) \chi_{\Omega_{-}^{\varepsilon}}$.

The present work is an extension of [10]. We shall prove the local existence of a unique solution to the free boundary problem (1.7) with $\varepsilon=0$, which we shall call the limit free boundary problem. Then we shall show that the solution of 
the limit free boundary problem approximates the solution of the system (1.1), (1.2). In order to prove this, we need to establish a theorem on the generation of the interface which is a refined version of the one given by Chen [10].

It is worth mentioning that there is a significant difference for the free boundary problem (1.7) between the case $\varepsilon>0$ and the case $\varepsilon=0$. The second equation in (1.7) is parabolic (in local coordinates) when $\varepsilon>0$, but, when $\varepsilon=0$, it is a Hamilton-Jacobi equation, a fully nonlinear first order PDE which requires stronger smoothness assumptions on the function $\mathscr{V}(v(x, t))$ in order to ensure a unique classical solution.

Our method for proving the uniqueness of the classical solution of the limit free boundary problem can be applied also to the free boundary problem (1.7) (for $\varepsilon>0$ ) to derive its uniqueness (existence was already established in [10]).

The special forms of $f$ and $g$ in (1.3) and (1.4) are introduced only for convenience. In fact, our method applies also to the case when $f=f(u, v, x, t)$ and $g=g(u, v, x, t)$. The essential assumption is that $f$ is bistable and that $f$ and $g$ are monotone in $v$ and $u$ respectively.

Our method also applies to the Cauchy problem, i.e., to the initial value problem (1.1), (1.2) with $\Omega=\mathscr{R}^{n}$.

The extension of our results to the case of an arbitrary time interval remains open. Although the Hamilton-Jacobi equations (e.g., the second equation in problem (1.7) with $\mathscr{V}(v(x, t))$ being a known function) have been extensively studied and many global existence results have been established $[4,11,17,29$, 32] (and the references therein), we cannot get the global asymptotic behavior of the system (1.1), (1.2) since our argument strongly relies on the regularity of the solution of the limit free boundary problem.

The plan of this paper is as follows. In $\S 2$ we formally derive the equations of the limit free boundary problem and state our main results. In $\S 3$ we establish the law of the generation of the interface (a weaker version of which has been established in [10]). Next, in $\S \S 4$ and 5, we shall establish the existence, uniqueness, and regularity of the solution of the limit free boundary problem. Finally in $\S 6$ we use a comparison lemma for the parabolic system (1.1), (1.2) to estimate the difference between the solution of the limit free boundary problem and the solution of (1.1), (1.2). The difference is of order $O(\varepsilon|\ln \varepsilon|)$ so that, as $\varepsilon \rightarrow 0$, the solution of (1.1), (1.2) tends to the solution of the limit free boundary problem.

Remark 1.1. After this paper was completed, the author was informed that Hilhorst, Nishiura, and Mimura [26] proved the existence of a unique solution to the limit free boundary problem (1.7) with $\varepsilon=0$ in the one-dimensional case, that Evans, Soner, and Souganidis [16] obtained the global asymptotic behavior of the solution of the Allen-Cahn equation, and that Giga, Goto, and Ishii [25] established the global existence of at least a weak solution to the free boundary problem (1.7) for both the case $\varepsilon>0$ and the case $\varepsilon=0$.

\section{Statement of the MAIN RESUlts}

Consider the second initial-boundary value problem:

$$
u_{t}^{\varepsilon}=\varepsilon \Delta u^{\varepsilon}+\frac{1}{\varepsilon} f\left(u^{\varepsilon}, v^{\varepsilon}\right) \quad \text { in } \Omega \times(0, T),
$$




$$
\begin{array}{ll}
v_{t}^{\varepsilon}=\Delta v^{\varepsilon}+g\left(u^{\varepsilon}, v^{\varepsilon}\right) & \text { in } \Omega \times(0, T), \\
u^{\varepsilon}(x, 0)=\varphi(x) & \text { for } x \in \Omega, \\
v^{\varepsilon}(x, 0)=\psi(x) & \text { for } x \in \Omega, \\
\partial_{n} u^{\varepsilon}=0 & \text { for }(x, t) \in \partial \Omega \times[0, T), \\
\partial_{n} v^{\varepsilon}=0 & \text { for }(x, t) \in \partial \Omega \times[0, T),
\end{array}
$$

where $\Omega$ is a smooth $\left(C^{3}\right)$ bounded domain in $\mathscr{R}^{n} \quad(n \geq 1), \partial_{n}$ is the normal derivative to $\partial \Omega$, and $T$ is any positive number. For simplicity, we shall assume that the functions $f$ and $g$ are given by (1.3) and (1.4).

For the sake of completeness, we first state a well-known existence and uniqueness result for the system (2.1)-(2.6).

Lemma 2.1. Assume that $\varphi$ and $\psi$ are in $C^{2}(\Omega)$ and satisfy the compatibility condition

$$
\partial_{n} \varphi=\partial_{n} \psi=0 \quad \forall x \in \partial \Omega .
$$

Then there exists a unique solution of the system (2.1)-(2.6) for all $0<T \leq+\infty$. Moreover, there exists a positive constant $C_{0}$ such that for all $\varepsilon>0$,

$$
\begin{gathered}
\left|u^{\varepsilon}(x, t)\right|+\left|v^{\varepsilon}(x, t)\right| \leq C_{0} \quad \forall x \in \Omega, t>0, \\
\left|v^{\varepsilon}(x, t)-\psi(x)\right| \leq C_{0} t \quad \forall x \in \Omega, t>0 .
\end{gathered}
$$

Proof. The existence of a unique solution follows from standard theory for parabolic systems whereas the estimate (2.8) follows by the invariant region theory (see, for example, Smoller [31, Chapter 14]). The estimate (2.9) follows by applying the comparison principle to the functions $v^{\varepsilon}$ and $\psi(x) \pm C_{0} t$ for the equation (2.2).

Observe that for smooth initial data $\psi$, the change of $v^{\varepsilon}$ in a short time is small, so that equation (2.1) is basically the same as the scalar equation (2.1) with $v^{\varepsilon}$ replaced by $\psi$. This observation leads to the following theorem.

Theorem 1 (Generation of interface). Assume that $\varphi$ and $\psi$ are in $C^{2}(\bar{\Omega})$ and satisfy the compatibility condition (2.7). Assume also that there exists a constant $\sigma>0$ such that

$$
|\psi(x)| \leq 2 \frac{\sqrt{3}}{9}-\sigma \quad \forall x \in \bar{\Omega} .
$$

Let $\left(u^{\varepsilon}, v^{\varepsilon}\right)$ be the unique solution of $(2.1)-(2.6)$. Then there exist positive constants $\varepsilon_{0}, \tau_{0}$, and $M_{0}$ such that for all $\varepsilon \in\left(0, \varepsilon_{0}\right], u^{\varepsilon}\left(x, \tau_{0} \varepsilon|\ln \varepsilon|\right)$ satisfies

$$
\begin{gathered}
h_{-}(\psi(x))-M_{0} \varepsilon|\ln \varepsilon| \leq u^{\varepsilon}\left(x, \tau_{0} \varepsilon|\ln \varepsilon|\right) \\
\leq h_{+}(\psi(x))+M_{0} \varepsilon|\ln \varepsilon| \quad \forall x \in \Omega,
\end{gathered}
$$

where

$$
\begin{aligned}
& \Omega_{M_{0} \varepsilon|\ln \varepsilon|}^{+}=\left\{x \in \Omega: \varphi(x) \geq h_{0}(\psi(x))+M_{0} \varepsilon|\ln \varepsilon|\right\}, \\
& \Omega_{M_{0} \varepsilon|\ln \varepsilon|}^{-}=\left\{x \in \Omega: \varphi(x) \leq h_{0}(\psi(x))-M_{0} \varepsilon|\ln \varepsilon|\right\} .
\end{aligned}
$$


Remark 2.1. As mentioned earlier, a weaker version of Theorem 1 was first proved in [10].

The proof, given in $\S 3$, is based on the method developed in [9] and involves the construction of supsolutions and subsolutions for equation (2.1).

We shall now formally find the asymptotic limit $(u, v)$ of the solution $\left(u^{\varepsilon}, v^{\varepsilon}\right)$ of $(2.1)-(2.6)$ as $\varepsilon \rightarrow 0^{+}$, leaving the rigorous proof to $\S 6$.

Denote by $\mathscr{D}_{T}$ the set $\mathscr{D} \times[0, T]$, where $\mathscr{D}$ is a set in $\mathscr{R}^{N}$. For $1 \leq p \leq$ $+\infty$, it is convenient to introduce the Sobolev norms

$$
\|f\|_{W_{p}^{k_{1}, k_{2}}\left(\mathscr{D}_{T}\right)} \equiv \sum_{i=1}^{k_{1}}\left\|D_{x}^{i} f\right\|_{L^{p}\left(\mathscr{D}_{T}\right)}+\sum_{i=0}^{k_{2}}\left\|D_{t}^{i} f\right\|_{L^{p}\left(\mathscr{D}_{T}\right)} \quad \forall k_{1}=1,2, k_{2}=0,1,
$$

and

$$
\|f\|_{W_{p}^{2,2}\left(\mathscr{D}_{T}\right)} \equiv \sum_{i=0}^{2} \sum_{j=0}^{2-i}\left\|D_{t}^{j} D_{x}^{i} f\right\|_{L^{p}\left(\mathscr{D}_{T}\right)} .
$$

Assuming that the assertion of Theorem 1 holds for each time $t>0$, we conclude that there exists a domain $D \equiv \bigcup_{0<t \leq T}\left(D^{t} \times\{t\}\right)$ such that

$$
u= \begin{cases}h_{+}(v) & \text { if }(x, t) \in D, \\ h_{-}(v) & \text { if }(x, t) \in \Omega_{T} \backslash \bar{D} .\end{cases}
$$

By the $L^{p} \quad(1<p<\infty)$ parabolic estimates, the $W_{p}^{2,1}\left(\bar{\Omega}_{T}\right)$ norm of $v^{\varepsilon}$ is uniformly bounded, so that its limit $v$ must be a (weak) solution of the equation

$$
v_{t}-\Delta v=g\left(h_{+}(v), v\right) \chi_{D}+g\left(h_{-}(v), v\right) \chi_{\Omega_{T} \backslash \bar{D}} \quad \forall(x, t) \in \Omega_{T} .
$$

Supplementing this parabolic equation with the initial and boundary data

$$
\begin{gathered}
v(x, 0)=\psi(x) \quad \forall x \in \Omega, \\
\partial_{n} v(x, t)=0 \quad \forall(x, t) \in \partial \Omega \times(0, T),
\end{gathered}
$$

we can solve for $v$ provided that $D$ is known.

To find the set $D$, we shall use the limit behavior of the solution $u^{\varepsilon}$ of $(2.1)$.

Denote by $\Gamma^{t}$ the (spatial) boundary of $D^{t}$; then (1.6) suggests that $D$ evolves in such a way that

$$
V_{\Gamma_{t}}=\mathscr{V}(v) \quad \forall x \in \Gamma^{t}, t \in[0, T],
$$

where $V_{\Gamma^{t}}$ is the inward normal velocity of $\Gamma^{t}$. Note that Theorem 1 implies

$$
\Gamma^{0} \equiv \partial D^{0}=\left\{x \in \bar{\Omega} \mid \varphi(x)-h_{0}(\psi(x))=0\right\} .
$$

Equations (2.15)-(2.19) form a free boundary problem which we shall refer to as the limit free boundary problem. We associate to $(v, \Gamma)$ a function $u$ defined by $(2.14)$ and refer to $(u, v, \Gamma)$ also as the solution of the limit free boundary problem.

Theorem 2. Assume that $\psi \in C^{2}(\bar{\Omega})$ and that $\Gamma^{0}$ is a $C^{2}$ hypersurface which is the boundary of a domain $D^{0} \subset \subset \Omega$. Then there exists a positive constant $T_{0}$ such that the limit free boundary problem (2.15)-(2.19) has a unique solution 
$(v, \Gamma)$ in the time interval $t \in\left[0, T_{0}\right]$, and the solution satisfies $v \in W_{\infty}^{2,1}\left(\bar{\Omega}_{T_{0}}\right)$ and $\Gamma \in W_{\infty}^{2,2}\left(\Gamma^{0} \times\left[0, T_{0}\right]\right)$.

To prove this theorem, we shall first study in $\S 4$ the solution $\Gamma$ of the problem (2.18), (2.19) where the function $\mathscr{V}(v)$ on the right-hand side of (2.18) is replaced by a given function $V \in W_{\infty}^{2,1}\left(\Omega_{T}\right)$. Then in $\S 5$ we shall establish the $W_{\infty}^{2,1}\left(\bar{\Omega}_{T}\right)$ regularity of the solution $v$ of the problem (2.15)-(2.17) where $D$ is a given domain compactly contained in $\Omega_{T}$ with a $C^{1+\alpha,(1+\alpha) / 2}$ lateral boundary. Denote by $\mathscr{H}$ the mapping which maps $\Gamma$ into the solution $v$ of (2.15)-(2.17) with $D$ being the (spatial) interior of $\Gamma$, and by $\mathscr{G}$ the mapping which maps $v$ into the solution $\Gamma$ of the problem (2.18), (2.19). We shall finally prove that the composition map $\mathscr{F}=\mathscr{G} \circ \mathscr{H}$ has a unique fixed point, thereby establishing the existence of a unique solution for the limit free boundary problem.

Finally in $\S 6$ we shall prove the following theorem.

Theorem 3 (Propagation of interface). Assume that the conditions of Theorems 1 and 2 hold, and that for some constant $c_{0}>0, \varphi$ and $\psi$ satisfy

$$
\begin{cases}\varphi(x)-h_{0}(\psi(x)) \geq c_{0}\left|\operatorname{dist}\left(x, \Gamma^{0}\right)\right| & \text { if } x \in D^{0}, \\ \varphi(x)-h_{0}(\psi(x)) \leq-c_{0}\left|\operatorname{dist}\left(x, \Gamma^{0}\right)\right| & \text { if } x \in \Omega \backslash D^{0},\end{cases}
$$

where $D^{0}$ is the set $\left\{x \in \Omega \mid \varphi(x)>h_{0}(\psi(x))\right\}$ which is also the interior of $\Gamma^{0}$, and $\operatorname{dist}\left(x, \Gamma^{0}\right)$ denotes the distance from $x$ to $\Gamma^{0}$. Then, there exist positive constants $\bar{M}_{0}$ and $\bar{\varepsilon}_{0}$ such that for all $\varepsilon \in\left(0, \bar{\varepsilon}_{0}\right]$ and $t \in\left[\tau_{0} \varepsilon|\ln \varepsilon|, T_{0}\right]$

$$
\left|u^{\varepsilon}(x, t)-u(x, t)\right| \leq \bar{M}_{0} \varepsilon|\ln \varepsilon| \quad \forall x \in\left\{x \in \Omega|| \operatorname{dist}\left(x, \Gamma^{t}\right)\left|>\bar{M}_{0} \varepsilon\right| \ln \varepsilon \mid\right\},
$$

where $\tau_{0}$ is as in Theorem 1, $T_{0}, v$, and $\Gamma$ are as in Theorem 2, and $u$ is given by (2.14).

Remark 2.2. If equation (2.1) is replaced by

$$
u_{t}-\Delta u=\frac{1}{\varepsilon^{2}}(F(u)-\varepsilon v),
$$

where $F$ is a bistable potential having equal depth of wells (i.e., $\mathscr{V}(0)=0$ ), then, formally, we have

$$
V_{\Gamma}=\frac{1}{\varepsilon}(\mathscr{V}(\varepsilon v)+\varepsilon K+o(\varepsilon))=\mathscr{V}^{\prime}(0) v+K+o(1) .
$$

In this case, if we replace the function $\mathscr{V}(v)+\varepsilon K$ on the right-hand side of the second equation in (1.7) by $\mathscr{V}^{\prime}(0) v+K$, then the existence of the resulting free boundary problem was established by Chen [10]. Using his existence and regularity theorem for this free boundary problem, we can rigorously prove (by the methods of [9] and of the present paper) a generation law which says that an interface generates in time $O\left(\varepsilon^{2}|\ln \varepsilon|\right)$, and a propagation law which says that the normal velocity of the interface is $\mathscr{V}^{\prime}(0) v+K$. 
In the sequel, we shall denote by the letter $C$ various positive constants which do not depend on $\varepsilon$.

\section{Generation OF interface}

Notice that (2.9) and (2.10) imply that there exists a positive constant $T_{1}$ such that

$$
\left|v^{\varepsilon}(x, t)\right| \leq \frac{2 \sqrt{3}}{9}-\frac{1}{2} \sigma \quad \forall(x, t) \in \Omega_{T_{1}}, \varepsilon \in(0,1) .
$$

In the sequel, we shall always assume that $T \leq T_{1}$ so that (3.1) holds, and therefore the three solutions $h_{-}(v), h_{0}(v)$, and $\bar{h}_{+}(v)$ of the algebraic equation $f(\cdot, v)=0$ are well defined.

In this section we shall show that in a short time of order $O(\varepsilon|\ln \varepsilon|)$ the solution $\left(u^{\varepsilon}, v^{\varepsilon}\right)$ of the system $(2.1)-(2.6)$ can be approximated by $(w(\varphi, t / \varepsilon ; \psi)$, $\psi)$, where $w(\xi, \tau ; v)$ is the solution of the ordinary differential equation

$$
\left\{\begin{array}{l}
w_{\tau}(\xi, \tau ; v)=f(w, v), \quad \tau>0 \\
w(\xi, 0 ; v)=\xi
\end{array}\right.
$$

with $\xi \in \mathscr{R}^{1}$ and $v \in\left[-2 \sqrt{3} / 9+\frac{1}{2} \sigma, 2 \sqrt{3} / 9-\frac{1}{2} \sigma\right]$ as parameters. To do this, we shall follow the ideas developed in [9]: First we construct a function $\tilde{w}(\xi, \tau ; b)$ which is the solution of the above ordinary differential equation with its right-hand side replaced by a modification $\tilde{f}$ of $f$; then we show that for some constant $M$ large enough, the function

$$
\underline{u}(x, t) \equiv \tilde{w}(\varphi(x)-M t, t / \varepsilon ; \psi(x)+M \varepsilon|\ln \varepsilon|)
$$

is a subsolution to the parabolic equation (2.1), and the function

$$
\bar{u}(x, t) \equiv \tilde{w}(\varphi(x)+M t, t / \varepsilon ; \psi(x)-M \varepsilon|\ln \varepsilon|)
$$

is a supersolution. ing:

In order to define $\tilde{f}(u, v)$, let $\rho(s) \in C_{0}^{\infty}\left(\mathscr{R}^{1}\right)$ be a cut-off function satisfy-

$$
\left\{\begin{aligned}
\rho(s)=1 & \text { if }|s| \leq 1, \\
\rho(s)=0 & \text { if }|s| \geq 2, \\
0<\rho(s)<1 & \text { if } 1<|s|<2, \\
-2 \leq s \rho^{\prime}(s) \leq 0 & \text { for all } s \in \mathscr{R}^{1}, \\
\left|\rho^{\prime \prime}(s)\right| \leq 4 & \text { for all } s \in \mathscr{R}^{1} .
\end{aligned}\right.
$$

Set

$$
\rho_{0} \equiv \rho\left(\frac{u-h_{0}(v)}{\varepsilon|\ln \varepsilon|}\right), \quad \rho_{+} \equiv \rho\left(\frac{u-h_{+}(v)}{\varepsilon|\ln \varepsilon|}\right), \quad \text { and } \quad \rho_{-} \equiv \rho\left(\frac{u-h_{-}(v)}{\varepsilon|\ln \varepsilon|}\right) \text {. }
$$

Define

$$
\begin{aligned}
\tilde{f}(u, v) \equiv & \rho_{0} \frac{u-h_{0}(v)}{|\ln \varepsilon|}+\rho_{+} \frac{h_{+}(v)-u}{|\ln \varepsilon|}+\rho_{-} \frac{h_{-}(v)-u}{|\ln \varepsilon|} \\
& +\left[1-\rho_{0}-\rho_{+}-\rho_{-}\right] f(u, v) .
\end{aligned}
$$


Notice that $F^{\prime}(u)=1-3 u^{2}$ vanishes only at $u= \pm \sqrt{3} / 3$; it is convenient to introduce a constant $\bar{\sigma}$ defined by

$\bar{\sigma} \equiv \min \left\{|\eta| \mid F\left(\frac{\sqrt{3}}{3}+\eta\right)=\frac{2 \sqrt{3}}{9}-\frac{1}{4} \sigma\right.$ or $\left.F\left(-\frac{\sqrt{3}}{3}+\eta\right)=-\frac{2 \sqrt{3}}{9}+\frac{1}{4} \sigma\right\}$.

Lemma 3.1. There exist positive constants $\bar{\varepsilon}$ and $C$ such that for all $\varepsilon \in(0, \bar{\varepsilon}]$ and $v \in\left[-2 \sqrt{3} / 9+\frac{1}{2} \sigma, 2 \sqrt{3} / 9-\frac{1}{2} \sigma\right]$, the function $\tilde{f}$ defined in (3.6) satisfies

$$
|\tilde{f}(u, v)-f(u, v)| \leq C \varepsilon|\ln \varepsilon| \quad \forall u \in\left[-C_{0}, C_{0}\right],
$$

$$
\begin{aligned}
\left|\tilde{f}_{u}(u, v)\right| \leq C & \forall u \in\left[-C_{0}, C_{0}\right], \\
\left|\tilde{f}_{v}(u, v)\right| \leq C & \forall u \in\left[-C_{0}, C_{0}\right], \\
\tilde{f}_{u}(u, v) \geq \frac{1}{|\ln \varepsilon|} & \forall u \in\left[-\frac{\sqrt{3}}{3}+\bar{\sigma}, \frac{\sqrt{3}}{3}-\bar{\sigma}\right], \\
\tilde{f}_{u}(u, v) \leq-\frac{1}{|\ln \varepsilon|} & \forall u \in\left[-C_{0},-\frac{\sqrt{3}}{3}-\bar{\sigma}\right] \\
\left|\tilde{f}_{v v}(u, v)\right| \leq \frac{C}{\varepsilon|\ln \varepsilon|} & \forall u \in\left[-C_{0}, C_{0}\right], \\
\left|\tilde{f}_{v}(u, v)\right| \leq C\left|\tilde{f}_{u}(u, v)\right| & \forall u \in\left[-C_{0}, C_{0}\right],\left|u \pm \frac{\sqrt{3}}{3}\right| \geq \bar{\sigma},
\end{aligned}
$$

where $C_{0}$ is as in Lemma 2.1.

Proof. It is enough to consider the case $u \in A(v)$, where

$$
\begin{aligned}
A(v) \equiv\left\{\eta|| \eta-h_{0}(v)|\leq 2 \varepsilon| \ln \varepsilon|,| \eta-h_{-}(v) \mid \leq\right. & 2 \varepsilon|\ln \varepsilon|, \\
& \text { or } \left.\left|\eta-h_{+}(v)\right| \leq 2 \varepsilon|\ln \varepsilon|\right\},
\end{aligned}
$$

since if $u \notin A(v)$, then $\tilde{f}=f$, and the lemma is obviously true. We can divide the case $u \in A(v)$ into three subcases: (i) $\left|u-h_{0}(v)\right| \leq 2 \varepsilon|\ln \varepsilon|$, (ii) $\left|u-h_{-}(v)\right| \leq 2 \varepsilon|\ln \varepsilon|$, and (iii) $\left|u-h_{+}(v)\right| \leq 2 \varepsilon|\ln \varepsilon|$.

Consider subcase (i):

$$
\left|u-h_{0}(v)\right| \leq 2 \varepsilon|\ln \varepsilon| .
$$

If $\varepsilon$ is small enough, then $\rho_{-}=\rho_{+}=0$ and

$$
\tilde{f}(u, v)=\rho_{0} \frac{u-h_{0}(v)}{|\ln \varepsilon|}+\left(1-\rho_{0}\right) f(u, v) .
$$

It follows that

$$
\begin{aligned}
|f(u, v)-\tilde{f}(u, v)| & =\left|\rho_{0}\left[\frac{u-h_{0}}{|\ln \varepsilon|}-f(u, v)\right]\right| \\
& =\rho_{0}\left|u-h_{0}\right|\left|\frac{1}{|\ln \varepsilon|}-\frac{f(u, v)-f\left(h_{0}, v\right)}{u-h_{0}}\right| \leq C \varepsilon|\ln \varepsilon|
\end{aligned}
$$

by (3.15) and the Lipschitz continuity of $f$. 
Differentiating (3.16) with respect to $u$ yields

$$
\tilde{f}_{u}(u, v)=\frac{\rho_{0}^{\prime}}{\varepsilon|\ln \varepsilon|}\left[\frac{u-h_{0}}{|\ln \varepsilon|}-f\right]+\frac{\rho_{0}}{|\ln \varepsilon|}+\left[1-\rho_{0}\right] f_{u}(u, v) .
$$

Inequality (3.9) thus follows by (3.15) and the boundedness of $\rho^{\prime}$ and $f_{u}$.

To prove (3.11), notice that $f_{u}(u, v)>1 /|\ln \varepsilon|$ for any $u \in[-\sqrt{3} / 3+$ $\bar{\sigma}, \sqrt{3} / 3-\bar{\sigma}]$ and $\varepsilon$ small enough; it follows that the function $\left(u-h_{0}\right) /|\ln \varepsilon|-$ $f(u, v)$ has the same sign as $h_{0}-u$. Since $\rho_{0}^{\prime}$ also has the same sign as $h_{0}-u$, the first term on the right-hand side of (3.17) is nonnegative. It then follows from (3.17) that

$$
\tilde{f}_{u}(u, v) \geq \frac{\rho_{0}}{|\ln \varepsilon|}+\left[1-\rho_{0}\right] f_{u}(u, v) \geq \frac{1}{|\ln \varepsilon|},
$$

i.e., (3.11) holds.

Differentiating (3.16) with respect to $v$, we get

$$
\tilde{f}_{v}(u, v)=-\frac{\rho_{0}^{\prime} h_{0}^{\prime}}{\varepsilon|\ln \varepsilon|}\left[\frac{u-h_{0}}{|\ln \varepsilon|}-f\right]-\frac{\rho_{0} h_{0}^{\prime}}{|\ln \varepsilon|}+\left[1-\rho_{0}\right] f_{v}(u, v) .
$$

Comparing this with equation (3.17), we find that (3.14) and (3.10) hold. Differentiating $\tilde{f}_{v}$ with respect to $v$ and using (3.15) and the boundedness of $\rho^{\prime \prime}$, we get

$$
\tilde{f}_{v v}=\frac{\rho_{0}^{\prime \prime} h_{0}^{\prime 2}}{(\varepsilon|\ln \varepsilon|)^{2}}\left[\frac{u-h_{0}}{|\ln \varepsilon|}-f\right]+\frac{1}{\varepsilon|\ln \varepsilon|}[\text { terms of order } O(1)]=O\left(\frac{1}{\varepsilon|\ln \varepsilon|}\right) .
$$

Therefore, Lemma 3.1 holds in subcase (i). Similarly, we can treat subcases (ii) and (iii) and therefore establish the assertion of the lemma.

We now define the function $\tilde{w}(\xi, \tau ; v)$ as the solution of the ordinary differential equation

$$
\left\{\begin{array}{l}
\tilde{w}_{\tau}(\xi, \tau ; v)=\tilde{f}(\tilde{w}, v), \quad \tau>0, \\
\tilde{w}(\xi, 0 ; v)=\xi,
\end{array}\right.
$$

where $\xi \in\left[-C_{0}, C_{0}\right]$ and $v \in\left[-2 \sqrt{3} / 9+\frac{1}{2} \sigma, 2 \sqrt{3} / 9-\frac{1}{2} \sigma\right]$ serve as parameters.

Some properties of the function $\tilde{w}(\xi, \tau ; v)$ are listed in the following lemma.

Lemma 3.2. Assume that $\xi \in\left[-C_{0}, C_{0}\right]$ and $v \in\left[-2 \sqrt{3} / 9+\frac{1}{2} \sigma, 2 \sqrt{3} / 9-\frac{1}{2} \sigma\right]$, and let $\tilde{w}(\xi, \tau ; v)$ be the solution of (3.18). Then

(1) $\tilde{w}(\xi, \tau ; v) \in C^{2}\left(\mathscr{R}^{1} \times \mathscr{R}^{+} \times\left[-2 \sqrt{3} / 9+\frac{1}{2} \sigma, 2 \sqrt{3} / 9-\frac{1}{2} \sigma\right]\right)$, and

$$
\tilde{w}_{\xi}(\xi, \tau ; v)>0 \quad \forall \tau>0 ;
$$

(2) there exist positive constants $\tau_{0}$ and $\varepsilon_{0}$ such that if $\varepsilon \in\left(0, \varepsilon_{0}\right]$ and $\tau \geq \tau_{0}|\ln \varepsilon|$, then $\tilde{w}(\xi, \tau ; v)$ satisfies

$$
\begin{gathered}
\tilde{w}(\xi, \tau ; v) \geq h_{+}(v)-2 \varepsilon|\ln \varepsilon| \quad \forall \xi \in\left[h_{0}(v)+2 \varepsilon|\ln \varepsilon|, \infty\right), \\
\tilde{w}(\xi, \tau ; v) \leq h_{-}(v)+2 \varepsilon|\ln \varepsilon| \quad \forall \xi \in\left(-\infty, h_{0}(v)-2 \varepsilon|\ln \varepsilon|\right],
\end{gathered}
$$

and

$$
h_{-}(v)-2 \varepsilon|\ln \varepsilon| \leq \tilde{w}(\xi, \tau ; v) \leq h_{+}(v)+2 \varepsilon|\ln \varepsilon| \quad \forall \xi \in\left[-C_{0}, C_{0}\right]
$$


(3) there exists a positive constant $C_{1}$ depending only on $\varepsilon_{0}$ and $\tau_{0}$ defined in (2) such that if $\varepsilon \in\left(0, \varepsilon_{0}\right]$ and $\tau \in\left[0, \tau_{0}|\ln \varepsilon|\right]$, then

$$
\begin{aligned}
\left|\tilde{w}_{\xi \xi}\right| & \leq C_{1} \tilde{w}_{\xi} / \varepsilon, \\
\left|\tilde{w}_{v}\right| & \leq C_{1}\left(1+\tilde{w}_{\xi}\right), \\
\left|\tilde{w}_{\xi v}\right| & \leq C_{1}\left(1+\tilde{w}_{\xi}\right) / \varepsilon, \\
\left|\tilde{w}_{v v}\right| & \leq C_{1}\left(1+\tilde{w}_{\xi}\right) / \varepsilon .
\end{aligned}
$$

Proof. Since $\tilde{w}_{\xi}$ satisfies $\left(\tilde{w}_{\xi}\right)_{\tau}=\tilde{f}_{u}(\tilde{w}, v) \tilde{w}_{\xi}$ and $\tilde{w}_{\xi}(\xi, 0 ; v)=1$, it follows that

$$
\tilde{w}_{\xi}(\xi, \tau ; v)=\exp \left(\int_{0}^{\tau} \tilde{f}_{u}\left(\tilde{w}\left(\xi, \tau_{1} ; v\right), v\right) d \tau_{1}\right) ;
$$

the first assertion of the lemma thus follows.

Notice that $u=h_{0}, u=h_{-}$, and $u=h_{+}$are the only solutions of the equation $\tilde{f}(u, v)=0$; it follows that $\tilde{f}(u, v)>0$ when $u<h_{-}$or $h_{0}<$ $u<h_{+}$, and $\tilde{f}(u, v)<0$ when $h_{-}<u<h_{0}$ or $u>h_{+}$. Consequently, as $\tau \nearrow+\infty$, we have

$$
\begin{aligned}
& \left|\tilde{w}(\xi, \tau ; v)-h_{-}(v)\right| \searrow 0 \text { for all } \xi<h_{0}(v), \\
& \left|\tilde{w}(\xi, \tau ; v)-h_{+}(v)\right| \searrow 0 \text { for all } \xi>h_{0}(v) .
\end{aligned}
$$

Since there exists a constant $\alpha>0$ such that

$$
f(u, v) \geq \alpha \min \left\{u-h_{0}, h_{+}-u\right\} \quad \forall u \in\left[h_{0}, h_{+}\right],
$$

by solving (3.18) we find that

$$
\tilde{w}\left(h_{0}+2 \varepsilon|\ln \varepsilon|, \tau ; v\right) \geq \min \left\{\frac{h_{0}+h_{+}}{2}, h_{0}+2 \varepsilon|\ln \varepsilon| e^{\alpha \tau}\right\}
$$

and

$$
\tilde{w}\left(\frac{h_{0}+h_{+}}{2}, \tau ; v\right) \geq h_{+}-\max \left\{2 \varepsilon|\ln \varepsilon|, \frac{h_{+}-h_{0}}{2} e^{-\alpha \tau}\right\} .
$$

It follows that

$$
\tilde{w}\left(h_{0}+2 \varepsilon|\ln \varepsilon|, \frac{\tau_{0}}{2}|\ln \varepsilon| ; v\right) \geq \frac{h_{0}+h_{+}}{2}
$$

and

$$
\tilde{w}\left(\frac{h_{0}+h_{+}}{2}, \frac{\tau_{0}}{2}|\ln \varepsilon| ; v\right) \geq h_{+}-2 \varepsilon|\ln \varepsilon|
$$

provided that we take $\tau_{0}=2 / \alpha$ and $\varepsilon$ small enough. Therefore, for all $\xi \geq$ $h_{0}+2 \varepsilon|\ln \varepsilon|$ and $\tau \geq \tau_{0}|\ln \varepsilon|$

$$
\begin{aligned}
\tilde{w}(\xi, \tau ; v) & \geq \tilde{w}\left(h_{0}+2 \varepsilon|\ln \varepsilon|, \tau ; v\right) \\
& \geq \tilde{w}\left(\frac{h_{0}+h_{+}}{2}, \tau-\frac{\tau_{0}}{2}|\ln \varepsilon| ; v\right) \geq h_{+}-2 \varepsilon|\ln \varepsilon|,
\end{aligned}
$$

i.e., inequality (3.20) holds. Similarly, we can show that (3.21) and (3.22) hold. The second assertion of the lemma thus follows.

To prove the last assertion of the lemma, we first consider the case

$$
\left|\xi-h_{0}(v)\right|<e^{-\tau_{0}} \varepsilon|\ln \varepsilon| .
$$


Since $\tilde{f}(u, v)=\left(u-h_{0}(v)\right) /|\ln \varepsilon|$ when $\left|u-h_{0}(v)\right| \leq \varepsilon|\ln \varepsilon|$, we can solve (3.18) explicitly to get

$$
\tilde{w}(\xi, \tau ; v)=h_{0}(v)+\left(\xi-h_{0}(v)\right) e^{\tau /|\ln \varepsilon|} \forall \tau \in\left[0, \tau_{0}|\ln \varepsilon|\right]
$$

Direct differentiation then yields

$$
\begin{aligned}
\tilde{w}_{\xi \xi}(\xi, \tau ; v) & =0, \\
\tilde{w}_{\xi v}(\xi, \tau ; v) & =0, \\
\tilde{w}_{v}(\xi, \tau ; v) & =h_{0}^{\prime}(v)\left[1-e^{\tau /|\ln \varepsilon|}\right] \\
\tilde{w}_{v v}(\xi, \tau ; v) & =h_{0}^{\prime \prime}(v)\left[1-e^{\tau /|\ln \varepsilon|}\right] .
\end{aligned}
$$

Assertion (3) thus holds in this case. Similarly, we can treat the cases $\mid \xi$ $h_{-}(v)|<\varepsilon| \ln \varepsilon \mid$ and $\left|\xi-h_{+}(v)\right|<\varepsilon|\ln \varepsilon|$.

It now remains to consider the case when $\xi \in B(v)$, where

$$
B(v) \equiv\left\{\eta \in\left[-C_{0}, C_{0}\right]|| \eta-h_{ \pm}(v)|\geq \varepsilon| \ln \varepsilon \mid \text { and }\left|\eta-h_{0}(v)\right| \geq e^{-\tau_{0}} \varepsilon|\ln \varepsilon|\right\} \text {. }
$$

We claim that

$$
|\tilde{f}(\tilde{w}(\xi, \tau ; v), v)| \geq e^{-\tau_{0}} \varepsilon \quad \forall \tau \in\left[0, \tau_{0}|\ln \varepsilon|\right], \quad \xi \in B(v) .
$$

In fact, one can easily verify that when $\xi \in\left(h_{0}, h_{+}\right)$, the function $\tilde{w}(\xi, \tau ; v)$ is monotone increasing in $\tau$ and $\xi$, and that the solution of (3.18) with $\xi=$ $h_{+}-\varepsilon|\ln \varepsilon|$ is given by

$$
\tilde{w}\left(h_{+}-\varepsilon|\ln \varepsilon|, \tau ; v\right)=h_{+}-\varepsilon|\ln \varepsilon| e^{-\tau /|\ln \varepsilon|} .
$$

Hence, when $\xi \in\left[h_{0}+e^{-\tau_{0}} \varepsilon|\ln \varepsilon|, h_{+}-\varepsilon|\ln \varepsilon|\right]$ and $\tau \in\left[0, \tau_{0} \varepsilon|\ln \varepsilon|\right]$, it follows that

$$
\xi \leq \tilde{w}(\xi, \tau ; v) \leq \tilde{w}\left(h_{+}-\varepsilon|\ln \varepsilon|, \tau_{0} \varepsilon|\ln \varepsilon| ; v\right)=h_{+}-e^{-\tau_{0}} \varepsilon|\ln \varepsilon|,
$$

and, by (3.12), inequality (3.29) holds for the case $\xi \in\left[h_{0}+e^{-\tau_{0}} \varepsilon|\ln \varepsilon|, h_{+}-\right.$ $\varepsilon|\ln \varepsilon|]$. Similarly, we can treat the rest of the case $\xi \in B(v)$, and therefore (3.29) holds.

We shall now show (3.23)-(3.26) for the case $\xi \in B(v)$. By the change of variables

$$
d \tau_{1} \equiv \frac{d \tilde{w}}{\tilde{w}_{\tau}\left(\xi, \tau_{1} ; v\right)}=\frac{d \tilde{w}}{\tilde{f}\left(\tilde{w}\left(\xi, \tau_{1} ; v\right), v\right)}
$$

for the integral on the right-hand side of equation (3.27), we get

$$
\tilde{w}_{\xi}(\xi, \tau ; v)=\exp \left(\left.\ln |f(z, v)|\right|_{\xi} ^{\tilde{w}(\xi, \tau ; v)}\right)=\frac{\tilde{f}(\tilde{w}(\xi, \tau ; v), v)}{\tilde{f}(\xi, v)}
$$

Differentiating this expression with respect to $\xi$ and taking absolute values, we get

$$
\begin{aligned}
\left|\tilde{w}_{\xi \xi}(\xi, \tau ; v)\right| & =\left|\frac{\tilde{f}_{u}(\tilde{w}, v)}{\tilde{f}(\xi, v)} \tilde{w}_{\xi}-\frac{\tilde{f}(\tilde{w}, v)}{\tilde{f}^{2}(\xi, v)} \tilde{f}_{u}(\xi, v)\right| \\
& =\left|\frac{\tilde{f}_{u}(\tilde{w}, v)}{\tilde{f}(\xi, v)} \tilde{w}_{\xi}-\frac{\tilde{f}_{u}(\xi, v)}{\tilde{f}(\xi, v)} \tilde{w}_{\xi}(\xi, v)\right| \leq \frac{C e^{\tau_{0}}}{\varepsilon} \tilde{w}_{\xi}
\end{aligned}
$$

by (3.29) and the boundedness of $\tilde{f}_{u}$; inequality (3.23) thus follows. 
Since $\tilde{w}_{v}(\xi, \tau ; v)$ satisfies $\left(\tilde{w}_{v}\right)_{\tau}=\tilde{f}_{u}(\tilde{w}, v) \tilde{w}_{v}+\tilde{f}_{v}(\tilde{w}, v)$ and $\tilde{w}_{v}(\xi, 0 ; v)$ $=0$, we have

$$
\tilde{w}_{v}(\xi, \tau ; v)=\int_{0}^{\tau} \exp \left(\int_{\tau_{1}}^{\tau} \tilde{f}_{u}\left(\tilde{w}\left(\xi, \tau_{2} ; v\right), v\right) d \tau_{2}\right) \tilde{f}_{v}\left(\tilde{w}\left(\xi, \tau_{1} ; v\right), v\right) d \tau_{1} .
$$

Applying the change of variables (3.30) to the integrals on the right-hand side yields

$$
\begin{aligned}
\tilde{w}_{v}(\xi, \tau ; v)= & \int_{0}^{\tau} \frac{\tilde{f}(\tilde{w}(\xi, \tau ; v), v)}{\tilde{f}\left(\tilde{w}\left(\xi, \tau_{1} ; v\right), v\right)} \tilde{f}_{v}\left(\tilde{w}\left(\xi, \tau_{1} ; v\right), v\right) d \tau_{1} \\
= & \tilde{f}(\tilde{w}, v) \int_{\xi}^{\tilde{w}} \frac{\tilde{f}_{v}(z, v)}{\tilde{f}^{2}(z, v)} d z \\
= & \tilde{f}(\tilde{w}, v) \int_{\xi_{|z \pm \sqrt{3} / 3| \leq \bar{\sigma}}}^{\tilde{w}} \frac{\tilde{f}_{v}(z, v)}{\tilde{f}^{2}(z)} d z \\
& +\tilde{f}(\tilde{w}, v) \int_{\xi_{|z \pm \sqrt{3} / 3| \geq \bar{\sigma}}}^{\frac{\tilde{w}}{\tilde{f}^{2}(z, v)}} d z .
\end{aligned}
$$

The first integral on the right-hand side of the last equality is bounded since $|\tilde{f}(z, v)| \geq \sigma / 4$ when $|z \pm \sqrt{3} / 3| \leq \bar{\sigma}$, by the definition of $\bar{\sigma}$ in (3.7). The last integral in (3.33) can be estimated by

$$
\begin{aligned}
& \left|\int_{\xi_{|z \pm \sqrt{3} / 3| \geq \bar{\sigma}}} \frac{\tilde{f}_{v}(z, v)}{\tilde{f}^{2}(z, v)} d z\right|=\left|\int_{\xi_{|z \pm \sqrt{3} / 3| \geq \bar{\sigma}}}^{\tilde{w}} \frac{\tilde{f}_{v}(z, v)}{\tilde{f}_{u}(z, v)} d\left(\frac{1}{\tilde{f}(z, v)}\right)\right| \\
& \quad \leq\left\{\sup _{|z \pm \sqrt{3} / 3| \geq \bar{\sigma}} \frac{\left|\tilde{f}_{v}(z, v)\right|}{\left|\tilde{f}_{u}(z, v)\right|}\right\}\left[\frac{1}{|\tilde{f}(\xi, v)|}+\frac{1}{|\tilde{f}(\tilde{w}, v)|}\right] \\
& \quad \leq C\left[\frac{1}{|\tilde{f}(\xi, v)|}+\frac{1}{|\tilde{f}(\tilde{w}, v)|}\right]
\end{aligned}
$$

by (3.14). It follows that

$$
\left|\tilde{w}_{v}(\xi, \tau ; v)\right| \leq C|\tilde{f}(\tilde{w}, v)|\left[1+\frac{1}{|\tilde{f}(\xi, v)|}+\frac{1}{|\tilde{f}(\tilde{w}, v)|}\right] \leq C\left(1+\tilde{w}_{\xi}\right),
$$

where we have used (3.31) in the last inequality. This establishes (3.24).

Differentiating (3.31) with respect to $v$ yields

$$
\tilde{w}_{\xi v}(\xi, \tau ; v)=\frac{\tilde{f}_{u}(\tilde{w}, v) \tilde{w}_{v}+\tilde{f}_{v}(\tilde{w}, v)}{\tilde{f}(\xi, v)}-\frac{\tilde{f}(\tilde{w}, v)}{\tilde{f}^{2}(\xi, v)} \tilde{f}_{v}(\xi, v) .
$$

The right-hand side is bounded by $C\left(1+\tilde{w}_{\xi}\right) / \varepsilon$ since $\tilde{f}_{u}$ and $\tilde{f}_{v}$ are bounded, $|\tilde{f}| \geq e^{-\tau_{0} \varepsilon}$ (by (3.29)), $\left|\tilde{w}_{v}\right| \leq C\left(1+\tilde{w}_{\xi}\right)$, and $\tilde{f}(\tilde{w}, v)=\tilde{w}_{\xi} \tilde{f}(\xi, v)$; inequality (3.25) thus follows.

It remains to prove (3.26). Differentiating the expression in (3.32) with respect to $v$ yields

$$
\begin{aligned}
\tilde{w}_{v v}= & {\left[\tilde{f}_{u}(\tilde{w}, v) \tilde{w}_{v}+\tilde{f}_{v}(\tilde{w}, v)\right] \int_{\xi}^{\tilde{w}} \frac{\tilde{f}_{v}(z, v)}{\tilde{f}^{2}(z, v)} d z } \\
& +\frac{\tilde{f}_{v}(\tilde{w}, v)}{\tilde{f}(\tilde{w}, v)} \tilde{w}_{v}+\tilde{f}(\tilde{w}, v) \int_{\xi}^{\tilde{w}}\left[\frac{\tilde{f}_{v v}(z, v)}{\tilde{f}^{2}(z, v)}-\frac{2 \tilde{f}_{v}^{2}(z, v)}{\tilde{f}^{3}(z, v)}\right] d z .
\end{aligned}
$$


From (3.33) and (3.34), we know that the first integral on the right-hand side is bounded by

$$
C\left(1+\frac{1}{|\tilde{f}(\xi, v)|}+\frac{1}{|\tilde{f}(\tilde{w}, v)|}\right) .
$$

Proceeding as in (3.33) and (3.34), we can also estimate

$$
\begin{aligned}
\left|\int_{\xi}^{\tilde{w}} \frac{\tilde{f}_{v v}}{\tilde{f}^{2}(z, v)} d z\right| & \leq \frac{C}{\varepsilon|\ln \varepsilon|}+\left\{\sup _{|z \pm \sqrt{3} / 3| \geq \bar{\sigma}} \frac{\left|\tilde{f}_{v v}\right|}{\left|\tilde{f}_{u}(z, v)\right|}\right\}\left[\frac{1}{|\tilde{f}(\xi, v)|}+\frac{1}{|\tilde{f}(\tilde{w}, v)|}\right] \\
& \leq C\left[\frac{1}{\varepsilon|\ln \varepsilon|}+\frac{1}{\varepsilon|\tilde{f}(\xi, v)|}+\frac{1}{\varepsilon|\tilde{f}(\tilde{w}, v)|}\right],
\end{aligned}
$$

where we have used (3.11)-(3.13). Similarly, we can estimate

$$
\left|\int_{\xi}^{\tilde{w}} \frac{\tilde{f}_{v}^{2}}{\tilde{f}^{3}(z, v)} d z\right| \leq C\left\{1+\frac{1}{\left|\tilde{f}^{2}(\xi, v)\right|}+\frac{1}{\left|\tilde{f}^{2}(\tilde{w}, v)\right|}\right\} .
$$

Substituting these estimates into (3.35) and using the boundedness of $\tilde{f}_{u}$ and $\tilde{f}_{v}$, we get

$$
\begin{aligned}
\left|\tilde{w}_{v v}\right| \leq & C\left[\left|\tilde{w}_{v}\right|+1\right]\left[1+\frac{1}{|\tilde{f}(\xi, v)|}+\frac{1}{\mid \tilde{f}(\tilde{w}, v)}\right]+\frac{C\left|\tilde{w}_{v}\right|}{|\tilde{f}(\tilde{w}, v)|} \\
& +C|\tilde{f}(\tilde{w}, v)|\left[\frac{1}{\varepsilon|\ln \varepsilon|}+\frac{1}{\varepsilon|\tilde{f}(\xi, v)|}\right. \\
& \left.\quad+\frac{1}{\varepsilon|\tilde{f}(\tilde{w}, v)|}+\frac{1}{\left|\tilde{f}^{2}(\xi, v)\right|}+\frac{1}{\left|\tilde{f}^{2}(\tilde{w}, v)\right|}\right] \\
\leq & C\left[1+\tilde{w}_{\xi}\right] \frac{e^{\tau_{0}}}{\varepsilon},
\end{aligned}
$$

since $\tilde{f}(\tilde{w}, v)=\tilde{w}_{\xi} \tilde{f}(\xi, v),\left|\tilde{w}_{v}\right| \leq C\left(1+\tilde{w}_{\xi}\right)$, and $|f| \geq e^{-\tau_{0}} \varepsilon$. This completes the proof of the lemma.

Proof of Theorem 1. By symmetry, it suffices to prove (2.12) and the first inequality in (2.11). To do this, we shall show that the function $\underline{u}$ defined in (3.3) is a subsolution to (2.1).

First we show that $\underline{u}$ satisfies the differential inequality

$$
\underline{u}_{t}-\varepsilon \Delta \underline{u}-\frac{1}{\varepsilon} f\left(\underline{u}, v^{\varepsilon}\right) \leq 0 \quad \forall t \in\left(0, \tau_{0} \varepsilon|\ln \varepsilon|\right] .
$$

Direct calculation yields

$$
\begin{aligned}
\varepsilon \Delta \underline{u} & =\varepsilon\left[\tilde{w}_{\xi} \Delta \varphi+\tilde{w}_{\xi \xi}|\nabla \varphi|^{2}+2 \tilde{w}_{\xi v} \nabla \varphi \cdot \nabla \psi+\tilde{w}_{v} \Delta \psi+\tilde{w}_{v v}|\nabla \psi|^{2}\right] \\
& \leq C_{1} \sup _{x \in \Omega}\left\{\varepsilon|\Delta \varphi|+\varepsilon|\Delta \psi|+|\nabla \varphi|^{2}+|\nabla \psi|^{2}\right\}\left[1+\tilde{w}_{\xi}\right] \\
& \equiv C_{1} A_{0}\left[1+\tilde{w}_{\xi}\right]
\end{aligned}
$$

by $(3.23)-(3.26)$, where

$$
A_{0} \equiv \sup _{x \in \Omega, 0<\varepsilon \leq \varepsilon_{0}}\left\{\varepsilon|\Delta \varphi|+\varepsilon|\Delta \psi|+|\nabla \varphi|^{2}+|\nabla \psi|^{2}\right\}
$$


One can also compute

$$
\begin{aligned}
\underline{u}_{t}-\frac{1}{\varepsilon} f\left(\underline{u}, v^{\varepsilon}\right)= & \frac{1}{\varepsilon} \tilde{w}_{\tau}-M \tilde{w}_{\xi}-\frac{1}{\varepsilon} f\left(\tilde{w}, v^{\varepsilon}\right) \\
= & \frac{1}{\varepsilon} \tilde{f}(\tilde{w}, \psi+M \varepsilon|\ln \varepsilon|)-M \tilde{w}_{\xi} \\
& -\frac{1}{\varepsilon}\left[f(\tilde{w}, \psi+M \varepsilon|\ln \varepsilon|)+\psi+M \varepsilon|\ln \varepsilon|-v^{\varepsilon}\right] \\
\leq & \frac{1}{\varepsilon}|\tilde{f}-f|+\frac{1}{\varepsilon}\left|v^{\varepsilon}-\psi\right|-M \tilde{w}_{\xi}-M|\ln \varepsilon| \\
\leq & -M \tilde{w}_{\xi}-\left[M-C-C_{0} \tau_{0}\right]|\ln \varepsilon| \quad \forall t \in\left(0, \tau_{0} \varepsilon|\ln \varepsilon|\right],
\end{aligned}
$$

where we have used the relations $\tilde{w}_{\tau}=\tilde{f},|\tilde{f}-f| \leq C \varepsilon|\ln \varepsilon|$ (by (3.8)), and $\left|v^{\varepsilon}-\psi\right| \leq C_{0} \tau_{0} \varepsilon|\ln \varepsilon|($ by $(2.9))$. Hence,

$$
\underline{u}_{t}-\varepsilon \Delta \underline{u}-\frac{1}{\varepsilon} f\left(\underline{u}, v^{\varepsilon}\right) \leq\left(C+C_{0} \tau_{0}+C_{1} A_{0}-M\right)|\ln \varepsilon|+\left(C_{1} A_{0}-M\right) \tilde{w}_{\xi} \leq 0
$$

if $M$ is large enough (recall that $\tilde{w}_{\xi}>0$ ). Inequality (3.36) thus follows.

Note that $\tilde{w}(\xi, 0 ; v)=\xi$ implies

$$
\underline{u}(x, 0)=\tilde{w}(\varphi(x), 0 ; \psi(x)+M \varepsilon|\ln \varepsilon|)=\varphi(x)=u^{\varepsilon}(x, 0) \quad \forall x \in \Omega,
$$

and the compatibility assumption (2.7) implies

$$
\partial_{n} \underline{u}(x, t)=\tilde{w}_{\xi} \partial_{n} \varphi(x)+\tilde{w}_{v} \partial_{n} \psi(x)=0=\partial_{n} u^{\varepsilon}(x, t) \quad \forall x \in \partial \Omega, t>0 .
$$

Hence, applying a comparison theorem for the parabolic equation (2.1) to the pair of functions $u^{\varepsilon}$ and $\underline{u}$ yields

$$
\underline{u}(x, t) \leq u^{\varepsilon}(x, t) \quad \forall x \in \Omega, t \in\left[0, \tau_{0} \varepsilon|\ln \varepsilon|\right] .
$$

Consequently,

$$
u^{\varepsilon}\left(x, \tau_{0} \varepsilon|\ln \varepsilon|\right) \geq \tilde{w}\left(\varphi(x)-M \tau_{0} \varepsilon|\ln \varepsilon|, \tau_{0}|\ln \varepsilon| ; \psi(x)+M \varepsilon|\ln \varepsilon|\right) .
$$

Using the first inequality in (3.22) and the Lipschitz continuity of the function $h_{-}$, we get

$$
\begin{aligned}
u^{\varepsilon}\left(x, \tau_{0} \varepsilon|\ln \varepsilon|\right) & \geq h_{-}(\psi(x)+M \varepsilon|\ln \varepsilon|)-2 \varepsilon|\ln \varepsilon| \\
& \geq h_{-}(\psi(x))-(c M+2) \varepsilon|\ln \varepsilon|,
\end{aligned}
$$

which yields the first inequality in (2.11) if we take $M$ large enough.

Substituting (3.20) into (3.38), we obtain

$u^{\varepsilon}\left(x, \tau_{0} \varepsilon|\ln \varepsilon|\right) \geq h_{+}(\psi(x)+M \varepsilon|\ln \varepsilon|)-2 \varepsilon|\ln \varepsilon| \geq h_{+}(\psi(x))-(c M+2) \varepsilon|\ln \varepsilon|$

provided

$$
\varphi(x)-M \tau_{0} \varepsilon|\ln \varepsilon|>h_{0}(\psi(x)+M \varepsilon|\ln \varepsilon|)+2 \varepsilon|\ln \varepsilon| .
$$

The last condition can be fulfilled if

$$
\varphi(x)>h_{0}(\psi(x))+C \varepsilon|\ln \varepsilon|
$$

for some constant $C$ large enough. This establishes (2.12). Theorem 1 thus follows.

Remark 3.1. By carefully checking the proof of Theorem 1, one finds that equation (2.2) is used only to derive the a priori estimate (2.9). Hence, equation (2.2) 
can be replaced by any equation which, when coupled with equations (2.1) and (2.3)-(2.6), forms a system admitting a solution satisfying (2.9). In particular, Theorem 1 remains true if equation (2.2) is replaced by the equation

$$
v^{\varepsilon}-\mu \Delta v^{\varepsilon}=g\left(u^{\varepsilon}, v^{\varepsilon}\right),
$$

where $\mu=\mu(\varepsilon)$ is any constant satisfying $0 \leq \mu \leq C$ for some positive constant $C$ independent of $\varepsilon$.

Remark 3.2. The condition $\varphi \in C^{2}$ in Theorem 1 can be replaced by the weaker condition that $\varphi$ is Lipschitz continuous. In fact, the only modification needed in the proof is to replace the function $\varphi$ in (3.3) by $\underline{\varphi}^{\varepsilon}$ which satisfies $\varphi-C \varepsilon|\ln \varepsilon| \leq \underline{\varphi}^{\varepsilon} \leq \varphi$ and $\varepsilon\left|\Delta \varphi^{\varepsilon}\right|+\left|\nabla \varphi^{\varepsilon}\right| \leq A_{0}$ (cf. (3.37)), and to replace $\varphi$ in (3.4) by $\bar{\varphi}^{\varepsilon}$ satisfying the corresponding conditions. Similarly, the function $\psi$ may be assumed to be only Lipschitz continuous.

\section{MOTION OF HYPERSURFACES}

Let $\Gamma^{0}$ be a $C^{2}$ compact hypersurface in $\mathscr{R}^{n}$ and assume that $\Gamma^{0}$ is the boundary of a domain $D^{0} \subset \subset \Omega$. We shall study the evolution of $\Gamma^{0}$ when at each time $t \geq 0$, it moves with an outward normal velocity $V$, where $V=$ $V(x, t)$ is a given function belonging to $W_{\infty}^{2,1}\left(\Omega_{T}\right)$.

Denote by $\Gamma^{t}$ the evolution of $\Gamma^{0}$ at time $t$ and assume that for $t \in[0, \delta)$ with $\delta$ sufficiently small, $\Gamma^{t}$ is a $C^{1}$ deformation of $\Gamma_{0}$. Then we can find a function $\Phi(x, t) \in C^{1}\left(\Omega_{\delta}\right)$ which, for all $t \in(0, \delta)$, satisfies

$$
\left\{\begin{array}{l}
\Gamma^{t}=\{x \in \Omega \mid \Phi(x, t)=0\}, \\
|\nabla \Phi(x, t)| \neq 0 \quad \forall x \in \Gamma^{t}, \\
\Phi(x, t)<0 \quad \forall x \in \partial \Omega .
\end{array}\right.
$$

Let $D^{t}$ be the interior of $\Gamma^{t}$; then $D^{t}=\{x \in \Omega \mid \Phi(x, t)>0\}$ and the outward normal velocity of $\Gamma^{t}$ is $\Phi_{t} /|\nabla \Phi|$. Hence, the evolution of $\Gamma^{t}$ can be described by

$$
\Phi_{t}(x, t)=|\nabla \Phi(x, t)| V(x, t) \quad \forall x \in \Gamma^{t}, t \in(0, \delta),
$$

with the initial conditions

$$
\Gamma^{0}=\{x \in \Omega \mid \Phi(x, 0)=0\}, \quad D^{0}=\{x \in \Omega \mid \Phi(x, 0)>0\} .
$$

Definition 4.1. A family of hypersurfaces $\left\{\Gamma^{t}\right\}_{0 \leq t<\delta}$ is called a solution of the motion problem if there exists a function $\Phi \in C^{1}\left(\Omega_{\delta}\right)$ satisfying (4.1)-(4.3), and

$$
\frac{\Phi_{t}-|\nabla \Phi| V}{\Phi} \in W_{\infty}^{1,0}\left(\Omega_{\delta}\right)
$$

Remark 4.1. the condition (4.4) is imposed to ensure uniqueness.

Remark 4.2. The level set approach for the motion of hypersurfaces was first used by Barles [4], Sethian [32], and others.

In the sequel, we shall denote by $\Gamma^{0}(h)$ the set $\left\{x \in \Omega \mid \operatorname{dist}\left(x, \Gamma^{0}\right)<h\right\}$, where $h>0$ is an arbitrary constant. 
Let $L \leq \frac{1}{2} \operatorname{dist}\left(\Gamma^{0}, \partial \Omega\right)$ be a small fixed positive constant and let $\Phi_{0}: \Gamma^{0}(L)$ $\rightarrow \mathscr{R}^{1}$ be a $C^{2}$ function such that

$$
\begin{gathered}
\Gamma^{0}=\left\{x \in \Gamma^{0}(L) \mid \Phi_{0}(x)=0\right\}, \\
D^{0} \cap \Gamma^{0}(L)=\left\{x \in \Gamma^{0}(L) \mid \Phi_{0}(x)>0\right\}, \\
\left|\nabla \Phi_{0}(x)\right|>0 \quad \forall x \in \overline{\Gamma^{0}(L)} .
\end{gathered}
$$

Given $V \in W_{\infty}^{2,1}\left(\Omega_{T}\right)$, we extend it to a $W_{\infty}^{2,1}$ function in $\mathscr{R}^{N} \times(0, T)$ and consider the following system of ordinary differential equations:

$$
\begin{aligned}
\frac{d}{d t} Y(x, t) & =-\frac{Q(x, t)}{|Q(x, t)|} V(Y(x, t), t), \\
\frac{d}{d t} Q(x, t) & =|Q(x, t)| \nabla V(Y(x, t), t), \\
Y(x, 0) & =x, \\
Q(x, 0) & =\frac{\nabla \Phi_{0}(x)}{\left|\nabla \Phi_{0}(x)\right|},
\end{aligned}
$$

where $x \in \Gamma^{0}(L)$ is considered as a parameter.

This system has a unique solution as long as $|Q|$ remains positive. Assume that $(Y, Q)$ is a solution of $(4.5)-(4.8)$ in some interval $0 \leq t<\delta$. Then by (4.6), $\left|\frac{d}{d t} Q\right| \leq C|Q|$, where $C=\|\nabla V\|_{L^{\infty}\left(\Omega_{T}\right)}$. Since $|Q(x, 0)|=1$, it follows that

$$
e^{-C t} \leq|Q(x, t)| \leq e^{C t} \quad \forall x \in \Gamma^{0}(L) .
$$

This a priori bound shows that the system (4.5)-(4.8) admits a unique solution for all $t \in[0, T]$.

Notice that when $x \in \Gamma^{0}, Q(x, 0)$ is equal to the unit inward normal of $\Gamma^{0}$ so that $Y(x, t)$ is independent of the choice of $\Phi_{0}(x)$. In addition, $Y(x, t)$ is independent of the extension of $V$ as long as $Y(x, t)$ remains in $\Omega$.

Set $Y(A, t)=\{Y(x, t) \mid x \in A\}$. We shall show that $\left\{Y\left(\Gamma_{0}, t\right)\right\}_{0 \leq t \leq \delta}$ is the unique solution to the motion problem provided that $\delta$ is small enough.

Define $t_{*}$ by

$$
\begin{aligned}
& t_{*} \equiv \sup \{t \in[0, T] \mid \text { there exists an } h \in(0, L) \text { such that for all } \tau \in[0, t], \\
& Y\left(\Gamma^{0}(h), \tau\right) \subset \Omega \text { and the mapping } Y(\cdot, \tau) \text { : } \\
&\left.\Gamma^{0}(h) \rightarrow Y\left(\Gamma^{0}(h), \tau\right) \text { is a Lipschitz homomorphism }\right\} .
\end{aligned}
$$

Here, a mapping is called a Lipschitz homomorphism if both the mapping and its inverse are Lipschitz continuous.

The following lemma shows that $t_{*}$ is strictly positive.

Lemma 4.1. There exists a positive constant $T_{2}$ depending only the $W_{\infty}^{2,1}\left(\Omega_{T}\right)$ norm of $V$ such that $Y\left(\Gamma^{0}(L), t\right) \subset \Omega$ and the mapping

$$
Y(\cdot, t): \Gamma^{0}(L) \mapsto Y\left(\Gamma^{0}(L), t\right)
$$

is a Lipschitz homomorphism for each $t \in\left[0, T_{2}\right]$. Moreover, $Y(x, t)$ is Lipschitz continuous in $x$ and $t$ in the domain $\Gamma^{0}(L) \times\left[0, T_{2}\right]$ and its inverse $Y^{-1}(y, t)$ is Lipschitz in $y$ and $t$ in the domain $\bigcup_{t \in\left[0, T_{2}\right]}\left(Y\left(\Gamma^{0}(L), t\right) \times\{t\}\right)$. Proof. Since the right-hand side of $(4.5)$ is bounded, $Y(x, t)$ is Lipschitz in $t$ so that $Y\left(\Gamma^{0}(L), t\right) \subset \Omega$ for any $t$ small enough. 
Denote by $F_{1}$ and $F_{2}$ the right-hand sides of (4.5) and (4.6) respectively. Differentiating (4.5) and (4.6) with respect to $x$, we get, for each $x \in \Gamma^{0}(L)$,

$$
\begin{aligned}
& \frac{d}{d t}\left(\begin{array}{l}
\partial Y / \partial x \\
\partial Q / \partial x
\end{array}\right)=\left(\begin{array}{ll}
\partial F_{1} / \partial Y & \partial F_{1} / \partial Q \\
\partial F_{2} / \partial Y & \partial F_{2} / \partial Q
\end{array}\right)\left(\begin{array}{l}
\partial Y / \partial x \\
\partial Q / \partial x
\end{array}\right) \\
& \equiv A(x, t)\left(\begin{array}{l}
\partial Y / \partial x \\
\partial Q / \partial x
\end{array}\right), \\
& \frac{\partial Y}{\partial x}(x, 0)\left.=I_{n} \quad \text { (the unit } n \times n \text { matrix }\right), \\
& \frac{\partial Q}{\partial x}(x, 0)=-\frac{\partial \vec{n}}{\partial x}(x) .
\end{aligned}
$$

Since $V \in W_{\infty}^{2,1}$ and $|Q|$ is strictly positive (by (4.9)), the matrix $A$ is bounded; hence,

$$
\left\|\frac{\partial Y}{\partial x}(x, t), \frac{\partial Q}{\partial x}(x, t)\right\| \leq C \quad \forall x \in \Gamma^{0}(L), t \in[0, T],
$$

where $C$ depends only on $T$ and the $W_{\infty}^{2,1}$ norm of $V$. Integrating the first $n$ equations in (4.11) and using the initial condition (4.12), we get

$$
\frac{\partial Y}{\partial x}(x, t)=I_{n}+\int_{0}^{t}\left(\frac{\partial F_{1}}{\partial Y} \frac{\partial Y}{\partial x}+\frac{\partial F_{1}}{\partial Q} \frac{\partial Q}{\partial x}\right)(x, \tau) d \tau,
$$

and therefore

$$
\left\|\frac{\partial Y}{\partial x}(x, t)-I_{n}\right\| \leq C t \quad \forall x \in \Gamma^{0}(L), t \in[0, T] .
$$

This estimate shows that $Y$ is a Lipschitz homomorphism for all $t$ small enough.

Implicit differentiation yields

$$
\frac{\partial Y^{-1}}{\partial t}(y, t)=-\left(\frac{\partial Y}{\partial x}\right)^{-1} \frac{d}{d t} Y=\left.\left(\frac{\partial Y}{\partial x}(x, t)\right)^{-1} \frac{Q(x, t)}{|Q(x, t)|} V(y, t)\right|_{x=Y^{-1}(y, t)},
$$

which implies that $Y^{-1}$ is Lipschitz in $t$. The assertion of the lemma follows.

Lemma 4.2. If $\left\{\Gamma^{t}\right\}_{0 \leq t<\delta}$ is a solution of the motion problem for some $\delta \in$ $\left(0, t_{*}\right)$, then it is given by

$$
\Gamma^{t}=Y\left(\Gamma^{0}, t\right) \quad \forall t \in(0, \delta) .
$$

Proof. Let $\Phi$ be the function in Definition 4.1 and set $f \equiv\left(\Phi_{t}-|\nabla \Phi| V\right) / \Phi$. Then $f \in W_{\infty}^{1,0}\left(\Omega_{\delta}\right)$ and

$$
\Phi_{t}(x, t)-|\nabla \Phi(x, t)| V(x, t)=\Phi(x, t) f(x, t) \quad \forall x \in \Omega, t \in(0, \delta) .
$$

This equation can be solved by the method of characteristics as follows (see, for example, Courant and Hilbert [12]): For every $x \in \Omega$, denote by $y=\tilde{Y}(x, t)$ the characteristic curve starting from $x$, and set $\Psi(x, t)=\Phi(\widetilde{Y}(x, t), t)$ and $P(x, t)=\nabla_{\widetilde{Y}} \Phi(\widetilde{Y}(x, t), t)$. Then the triple $(\widetilde{Y}, \Psi, P)$ satisfies the following 
differential system:

$$
\begin{aligned}
\frac{d}{d t} \tilde{Y}(x, t) & =-\frac{P}{|P|} V(\tilde{Y}, t), \\
\frac{d}{d t} \Psi(x, t) & =\Psi f(\tilde{Y}, t), \\
\frac{d}{d t} P(x, t) & =f(\tilde{Y}, t) P+|P| \nabla V(\tilde{Y}, t)+\Psi \nabla f(X, t), \\
\widetilde{Y}(x, 0) & =x, \\
\Psi(x, 0) & =\Phi(x, 0), \\
P(x, 0) & =\nabla \Phi(x, 0) .
\end{aligned}
$$

The solution $\Psi$ of (4.17), (4.20) can be integrated:

$$
\Psi(x, t)=\Phi(x, 0) \exp \left(\int_{0}^{t} f(\tilde{Y}(x, \tau), \tau) d \tau\right)
$$

It follows that $\Psi(x, t)=0$ if and only if $\Phi(x, 0)=0$, i.e., if and only if $x \in \Gamma^{0}$. Therefore, we have

$$
\Gamma_{t} \equiv\{y \in \Omega \mid \Phi(y, t)=0\}=\left\{\tilde{Y}(x, t) \mid \Psi(x, t)=\tilde{Y}\left(\Gamma^{0}, t\right)\right\} .
$$

One can verify that when $x \in \Gamma^{0}$ (so that $\left.\Psi(x, t) \equiv 0\right)$, the pair $(\tilde{Y}(x, t)$, $\widetilde{Q}(x, t))$, where

$$
\widetilde{Q}(x, t) \equiv \frac{\exp \left(-\int_{0}^{t} f(\tilde{Y}(x, \tau), \tau) d \tau\right)}{|P(x, 0)|} P(x, t),
$$

forms a solution of (4.5)-(4.8); hence, by uniqueness, $\tilde{Y}(x, t)=Y(x, t)$ for all $X \in \Gamma^{0}$. The assertion of the lemma then follows from (4.22).

Theorem 4.3. Let $V \in W_{\infty}^{2,1}(\Omega \times(0, T))$ be given and define $t_{*}$ as in (4.10). Then, for any $\delta \in\left(0, t_{*}\right)$, the motion problem admits a unique solution in the time interval $[0, \delta]$.

Proof. In view of Lemma 4.2 , we need only show that $\left\{Y\left(\Gamma^{0}, t\right)\right\}_{0 \leq t \leq \delta}$ is a solution of the motion problem.

By the definition of $t_{*}$, there exists a positive constant $h$ depending on $\delta$ such that $Y\left(\Gamma^{0}(h), t\right) \subset \Omega$ and the mapping $Y(\cdot, t): \Gamma^{0}(h) \mapsto Y\left(\Gamma^{0}(h), t\right)$ is a Lipschitz homomorphism for all $t \in[0, \delta]$; thus the function $\widetilde{\Phi}$ defined by

$$
\tilde{\Phi}(y, t)=\Phi_{0}\left(Y^{-1}(x, t)\right)
$$

is Lipschitz continuous in both $y$ and $t$ in $\bigcup_{0 \leq t \leq \delta}\left(Y\left(\Gamma^{0}(h), t\right) \times\{t\}\right)$.

We claim that

$$
Q(x, t)=\frac{\nabla_{Y} \tilde{\Phi}(Y(x, t), t)}{\left|\nabla \Phi_{0}(x)\right|} \quad \forall x \in \Gamma^{0}(h), t \in[0, \delta] .
$$


To prove this, we compute

$$
\begin{aligned}
\frac{d}{d t}( & \left.Q(x, t) \cdot \frac{\partial Y}{\partial x}(x, t)\right)=\frac{d}{d t} Q \cdot \frac{\partial Y}{\partial x}+Q \cdot \frac{\partial}{\partial x}\left(\frac{d}{d t} Y\right) \\
& =|Q| \nabla V \cdot \frac{\partial Y}{\partial x}-Q \cdot \frac{\partial}{\partial x}\left(\frac{Q}{|Q|} V\right) \quad(\text { by }(4.5) \text { and }(4.6)) \\
& =|Q| \nabla V \cdot \frac{\partial Y}{\partial x}-Q \cdot \frac{\partial}{\partial x}\left(\frac{Q}{|Q|}\right) V-Q \cdot \frac{Q}{|Q|}\left(\nabla V \cdot \frac{\partial Y}{\partial x}\right) \\
& =-Q \frac{\partial}{\partial x}\left(\frac{Q}{|Q|}\right) V=-\frac{1}{2}|Q| \frac{\partial}{\partial x}\left(\left|\frac{Q}{|Q|}\right|^{2}\right) V=0 ;
\end{aligned}
$$

hence,

$$
Q(x, t) \cdot \frac{\partial Y}{\partial x}(x, t)=Q(x, 0) \cdot \frac{\partial Y}{\partial x}(x, 0)=\frac{\nabla \Phi_{0}(x)}{\left|\nabla \Phi_{0}(x)\right|} \quad \forall x \in \Gamma^{0}(h) .
$$

On the other hand, the definition of $\tilde{\Phi}$ implies that

$$
\tilde{\Phi}(Y(x, t), t)=\Phi_{0}(x) .
$$

Differentiating this identity with respect to $x$ yields

$$
\nabla \tilde{\Phi}(Y(x, t), t) \cdot \frac{\partial Y}{\partial x}(x, t)=\nabla \Phi_{0}(x) \quad \forall x \in \Gamma^{0}(h) .
$$

Dividing both sides by $\left|\nabla \Phi_{0}\right|$ and subtracting the resulting equation from (4.24) yields

$$
\left(Q-\frac{\nabla \tilde{\Phi}}{\left|\nabla \Phi_{0}\right|}\right) \frac{\partial Y}{\partial x}=0 .
$$

Since the matrix $\frac{\partial Y}{\partial x}$ is nondegenerate, the assertion (4.23) follows.

Differentiating (4.25) with respect to $t$, we get, for all $x \in \Gamma^{0}(h)$ and $t \in$ $[0, \delta]$,

$$
0=\nabla \tilde{\boldsymbol{\Phi}} \cdot \frac{d}{d t} Y+\tilde{\Phi}_{t}=-\nabla \tilde{\boldsymbol{\Phi}}(y, t) \cdot \frac{Q(y, t)}{|Q(y, t)|} V(y, t)+\left.\tilde{\Phi}_{t}(y, t)\right|_{y=Y(x, t)} ;
$$

using (4.23), we obtain

$$
\tilde{\Phi}_{t}(y, t)=|\nabla \tilde{\Phi}(y, t)| V(y, t) \quad \forall y \in Y\left(\Gamma^{0}(h), t\right), t \in[0, \delta] .
$$

From (4.23) and the fact that $Y^{-1}$ is Lipschitz in $y$ and $t$ and that $Q$ is Lipschitz in $x$ and $t$, we deduce that $\nabla \tilde{\Phi}$ is Lipschitz in $y$ and $t$ in the domain $\bigcup_{t \in[0, \delta]}\left(Y\left(\Gamma^{0}(h), t\right) \times\{t\}\right)$; by (4.26), $\tilde{\Phi}_{t}$ is also Lipschitz in $y$ and $t$. Hence, $\tilde{\Phi} \in W_{\infty}^{2,2}\left(\bigcup_{t \in[0, \delta]}\left(Y\left(\Gamma^{0}(h), t\right) \times\{t\}\right)\right)$.

To prove the theorem, we have to construct a function $\Phi$ satisfying Definition 4.1. We shall now construct it from $\tilde{\Phi}$.

Let $a$ be the minimum value of $\left|\Phi_{0}\right|$ on $\partial \Gamma^{0}(h)$ and let $G(\cdot): \mathscr{R}^{1} \mapsto \mathscr{R}^{1}$ be a $C^{2}$ function satisfying

$$
\begin{aligned}
& G(0)=0, \quad G^{\prime}(0)>0, \\
& G^{\prime}(s) \geq 0 \quad \forall s \in \mathscr{R}^{1}, \\
& G^{\prime}(s)=0 \quad \forall s \in(-\infty,-a / 2] \cup[a / 2, \infty) .
\end{aligned}
$$


Set

$$
\Phi(x, t) \equiv\left\{\begin{aligned}
G(a / 2) & \text { if } x \text { is in the inside of } Y\left(\Gamma^{0}(a), t\right), \\
G(\tilde{\Phi}(x, t)) & \text { if } x \in Y\left(\Gamma^{0}(a), t\right), \\
G(-a / 2) & \text { if } x \text { is on the outside of } Y\left(\Gamma^{0}(a), t\right) .
\end{aligned}\right.
$$

Clearly, $\Phi$ belongs to $W_{\infty}^{2,2}\left(\Omega_{\delta}\right)$ and it satisfies (4.1), (4.3). We can compute

$$
\begin{aligned}
\Phi_{t}-|\nabla \Phi| V=G^{\prime} \tilde{\Phi}_{t}-\left|G^{\prime} \nabla \tilde{\Phi}\right| V=\left[\tilde{\Phi}_{t}-|\nabla \tilde{\Phi}| V\right] G^{\prime}(\tilde{\Phi})=0 \\
\forall(x, t) \in \Omega_{\delta}
\end{aligned}
$$

by (4.26) and (4.27). The last equation shows that $\Phi$ satisfies (4.2) and (4.4); therefore, the family of the sets given by $(4.15)$ is a solution of the motion problem. This completes the proof of the theorem.

Next we establish some estimates for the solution of the motion problem. It is convenient to introduce a mapping $x_{0}(\eta, l): \Gamma^{0} \times(-h, h) \rightarrow \mathscr{R}^{N}$ defined by

$$
x_{0}(\eta, l) \equiv \eta+l \vec{n}(\eta) \quad \forall(\eta, l) \in \Gamma^{0} \times\left(-L_{0}, L_{0}\right)
$$

where $\vec{n}(\eta)$ is the unit outward normal to $\Gamma^{0}$ and $L_{0} \in\left(0, \frac{1}{2} \operatorname{dist}\left(\Gamma^{0}, \partial \Omega\right)\right)$ is a constant small enough such that $x_{0}$ is a local diffeomorphism.

Theorem 4.4. There exist a monotone increasing function $K(M)>0$ and $a$ monotone decreasing function $T(M)>0$ such that if

$$
\|V\|_{W_{\infty}^{2,1}\left(\bar{\Omega}_{T(M)}\right)} \leq M
$$

then the motion problem admits a unique solution in $[0, T(M)]$ and the solution is given by

$$
\Gamma^{t}=\left\{x_{0}(\eta, l(\eta, t)) \mid \eta \in \Gamma^{0}\right\} \quad \forall t \in[0, T(M)],
$$

where $l(\eta, t): \Gamma^{0} \times[0, T(M)] \mapsto\left(-L_{0}, L_{0}\right)$ is a function satisfying

$$
\begin{gathered}
\|l\|_{W_{\infty}^{2,2}\left(\Gamma^{0} \times[0, T(M)]\right)} \leq K(M), \\
\|l\|_{C^{3 / 2}\left(\Gamma^{0} \times[0, t]\right)} \leq K(M) t^{1 / 4} \quad \forall t \in[0, T(M)] .
\end{gathered}
$$

Proof. For each $\eta \in \Gamma_{0}$, consider the equation, for $l$,

$$
\Phi\left(x_{0}(\eta, l), t\right)=0
$$

where $\Phi$ is the function constructed in the proof of Theorem 4.3. Since $\Phi\left(x_{0}(\eta, 0), 0\right)=0$ and

$$
\begin{aligned}
\frac{\partial \Phi}{\partial l}\left(x_{0}(\eta, 0), 0\right) & =\nabla \Phi(\eta, 0) \cdot \frac{\partial x_{0}}{\partial l}(\eta, 0)=-G^{\prime}(0) \nabla \Phi_{0}(\eta) \cdot \vec{n}(\eta) \\
& =-G^{\prime}(0)\left|\nabla \Phi_{0}(\eta)\right| \neq 0,
\end{aligned}
$$

the implicit function theorem ensures that equation (4.34) defines a unique function $l=l(\eta, t)$ for $t$ small enough. Recalling that $\Phi \in W_{\infty}^{2,2}$, we deduce that $l \in W_{\infty}^{2,2}$, and therefore (4.32) follows.

Noting that $l(\eta, 0)=0 \forall \eta \in \Gamma^{0}$, we can use the mean value theorem and (4.32) to deduce that $\|l\|_{L^{\infty}\left(\Gamma^{0} \times[0, t]\right)} \leq C t$ for all $t \in[0, T(M)]$. Hence, by the interpolation

$$
\|\cdot\|_{C^{3 / 2}} \leq C\left(\|\cdot\|_{W_{\infty}^{2,2}}\right)^{3 / 4}\left(\|\cdot\|_{L^{\infty}}\right)^{1 / 4},
$$

inequality (4.33) follows; this completes the proof of the theorem. 
In the sequel, we shall denote by $\mathscr{G}$ the mapping from $v \in W_{\infty}^{2,1}\left(\bar{\Omega}_{T}\right)$ to $l$, the function in (4.31) where $\Gamma$ is the solution of the motion problem with $V=-\mathscr{V}(v)$.

Theorem 4.5. Let $v^{1}$ and $v^{2}$ be two functions in $W_{\infty}^{2,1}\left(\Omega_{T}\right)$, and set $l^{1}=$ $\mathscr{G} v^{1}$ and $l^{2}=\mathscr{G} v^{2}$. Then there exists a constant $C$ depending only on $M \equiv$ $\left\|v^{1}\right\|_{W_{\infty}^{2,1}\left(\Omega_{T}\right)}+\left\|v^{2}\right\|_{W_{\infty}^{2,1}\left(\Omega_{T}\right)}$, such that

$$
\left\|l^{1}-l^{2}\right\|_{C^{1}\left(\Gamma^{0} \times[0, t]\right)} \leq C t\left\|v^{1}-v^{2}\right\|_{W_{\infty}^{1,0}\left(\Omega_{T(M)}\right)} \quad \forall t \in[0, T(M)] .
$$

Proof. Denote by $\left(Y^{i}(x, t), Q^{i}(x, t)\right)$ the solution of the system (4.5)-(4.8) corresponding to $V^{i}=-\mathscr{V}\left(v^{i}\right)$. Then

$$
\begin{aligned}
\mid \frac{d}{d t}( & \left.Y^{1}-Y^{2}\right)|+| \frac{d}{d t}\left(Q^{1}-Q^{2}\right) \mid \\
\quad=\left|F_{1}\left(Y^{1}, Q^{1}\right)-F_{1}\left(Y^{2}, Q^{2}\right)\right|+\mid F_{2}\left(Y^{1}, Q^{1}\right)- & F_{2}\left(Y^{2}, Q^{2}\right) \mid \\
& \leq C\left[\left|Y^{1}-Y^{2}\right|+\left|Q^{1}-Q^{2}\right|+\left|\nabla V^{1}\left(Y^{1}, t\right)-\nabla V^{2}\left(Y^{2}, t\right)\right|\right. \\
& \left.\quad+\left|V^{1}\left(Y^{1}, t\right)-V^{2}\left(Y^{2}, t\right)\right|\right] \\
& \leq C\left[\left(1+\left\|V^{1}\right\|_{W_{\infty}^{2,0}\left(\Omega_{T(M)}\right)}\right)\left|Y^{1}-Y^{2}\right|+\left|Q^{1}-Q^{2}\right|+\left\|V^{1}-V^{2}\right\|_{W_{\infty}^{1,0}\left(\Omega_{T(M)}\right)}\right] .
\end{aligned}
$$

Since $Y^{1}=Y^{2}$ and $Q^{1}=Q^{2}$ at $t=0$, we can apply Gronwall's inequality to get

$$
\left|Y^{1}-Y^{2}\right|+\left|Q^{1}-Q^{2}\right| \leq C t\left\|V^{1}-V^{2}\right\|_{W_{\infty}^{1,0}\left(\Omega_{T(M)}\right)} .
$$

Denote by $Y^{i^{-1}}$ the inverse of $Y^{i}$. The identity $Y^{i}\left(Y^{i^{-1}}(y, t), t\right)=y \quad(i=$ $1,2)$ implies

$$
Y^{1}\left(Y^{2^{-1}}, t\right)-Y^{1}\left(Y^{1^{-1}}, t\right)=Y^{1}\left(Y^{2^{-1}}, t\right)-Y^{2}\left(Y^{2^{-1}}, t\right) .
$$

Applying the mean value theorem to the expression on the left-hand side and using (4.14), we get

$$
\left|Y^{1}\left(Y^{2^{-1}}, t\right)-Y^{1}\left(Y^{1^{-1}}, t\right)\right| \geq \frac{1}{2}\left|Y^{2^{-1}}-Y^{1^{-1}}\right|
$$

if $t \mathrm{y}$ is small enough; therefore, after using (4.37) to estimate the right-hand side of (4.38), we obtain

$$
\left|Y^{1^{-1}}-Y^{2^{-1}}\right| \leq C t\left\|V^{1}-V^{2}\right\|_{W_{\infty}^{1,0}\left(\Omega_{T(M)}\right)} .
$$

Denote by $\Phi^{i}(i=1,2)$ the function $\Phi$ constructed in the proof of Theorem 4.3 with $V=V^{i}$. Applying the estimates (4.37) and (4.39) in (4.23) and using (4.28), we find that

$$
\left\|\nabla \Phi^{1}-\nabla \Phi^{2}\right\|_{L^{\infty}\left(\Omega_{t}\right)}+\left\|\Phi_{t}^{1}-\Phi_{t}^{2}\right\|_{L^{\infty}\left(\Omega_{t}\right)} \leq C t\left\|V^{1}-V^{2}\right\|_{W_{\infty}^{1,0}\left(\Omega_{T(M)}\right)} .
$$

Since $\Phi^{1}(\cdot, 0)=\Phi^{2}(\cdot, 0)$, we can use the mean value theorem and the last estimate to conclude that

$$
\left\|\Phi^{1}-\Phi^{2}\right\|_{L^{\infty}\left(\Omega_{t}\right)} \leq C t\left\|V^{1}-V^{2}\right\|_{W_{\infty}^{1.0}\left(\Omega_{T(M)}\right)} .
$$


Recall that the function $l$ is defined implicitly by (4.34). By an argument similar to that which leads to (4.39), we can deduce from (4.40) and (4.41) that

$$
\left\|l^{1}-l^{2}\right\|_{C^{1}\left(\Gamma^{0} \times[0, t]\right)} \leq C t\left\|V^{1}-V^{2}\right\|_{W_{\infty}^{1,0}\left(\Omega_{T(M)}\right)} \quad \forall t \in[0, T(M)] .
$$

Since $V^{i}=-\mathscr{V}\left(v^{i}\right)$ and the function $\mathscr{V}$ is smooth, the assertion of the theorem follows.

\section{A $W_{\infty}^{2,1}$ ESTIMATE FOR A PARABOLIC EQUATION AND THE PROOF OF THEOREM 2}

Consider the parabolic problem

$$
\begin{aligned}
v_{t}-\Delta v & =f_{1}(v) \chi_{D}+f_{2}(v) \chi_{D^{c}}, & & x \in \Omega, t \in(0, T), \\
v(x, 0) & =\psi(x), & & x \in \Omega, \\
\partial_{n} v(x, t) & =0, & & x \in \partial \Omega, t \in(0, T),
\end{aligned}
$$

where $f_{1}(\cdot)$ and $f_{2}(\cdot)$ are Lipschitz continuous functions, $D \equiv \bigcup_{0 \leq t \leq T}\left(D^{t} \times\{t\}\right)$ is a domain compactly contained in $\Omega \times[0, T]$, and $D^{c}=\Omega \times[0, T] \backslash D$. For every $t \in[0, T]$, let $\Gamma^{t}=\partial D^{t}$ and set $\Gamma=\bigcup_{0<t<T}\left(\Gamma^{t} \times\{t\}\right)$. We assume that there exists a function $l: \Gamma_{0} \times[0, T] \rightarrow\left(-L_{0}, L_{0}\right)$ such that

$$
\Gamma^{t}=\left\{x_{0}(\eta, l(\eta, t)) \mid \eta \in \Gamma^{0}\right\} \quad \forall t \in[0, T],
$$

where $x_{0}$ is the mapping defined in (4.29) and $L_{0}$ is a small constant such that $x_{0}$ is a $C^{2}$ diffeomorphism (in $\eta$ and $l$ ) and $L_{0} \leq \frac{1}{2} \operatorname{dist}\left(\Gamma_{0}, \partial \Omega\right)$.

By the $L^{p}$ parabolic estimate (cf. Ladyzhenskaja et al. [27]), for any $p \in$ $(1, \infty)$, there exists a constant $C_{p}$ depending only on $\Omega$ and $T$ such that

$$
\|v\|_{W_{p}^{2,1}\left(\bar{\Omega}_{T}\right)} \leq C_{p}\left[\|\psi\|_{W_{p}^{2}(\bar{\Omega})}+\left\|f_{1}(v) \chi_{D}+f_{2}(v) \chi_{D^{c}}\right\|_{L^{p}\left(\bar{\Omega}_{T}\right)}\right]
$$

A sharper regularity result is established in the following theorem.

Theorem 5.1. Assume that $f_{1}$ and $f_{2}$ are uniformly Lipschitz continuous, and that $\psi \in C^{2}(\bar{\Omega})$ satisfies the compatibility condition (2.7). Then the problem (5.1)-(5.3) has a unique solution $v$ and it satisfies

$$
\|v\|_{W_{\infty}^{2,1}\left(\bar{\Omega}_{T}\right)} \leq C_{\alpha}\left(1+\|l\|_{C^{1+\alpha,(1+\alpha) / 2}\left(\Gamma^{0} \times[0, T]\right)}\right) \quad \forall \alpha \in(0,1)
$$

for some constant $C_{\alpha}$ independent of $l$.

Proof. Let

$$
\Gamma(x-\xi, t-\tau)=\frac{c_{n}}{(t-\tau)^{n / 2}} e^{-|x-\xi|^{2} / 4(t-\tau)}
$$

be the fundamental solution of the heat operator, and let $G(x, t ; \xi, \tau)=$ $\Gamma(x-\xi, t-\tau)+H(x, t ; \xi, \tau)$ be the Green function, where $H$ is a "regular" term which makes $G$ satisfy the boundary condition $\partial_{n} G=0$. In terms of Green's formula, $v \in C^{0}\left(\Omega_{T}\right)$ is a solution of (5.1)-(5.3) if and only if $v$ is a fixed point of $\mathscr{K}$, where

$$
\begin{aligned}
\mathscr{K}(v) \equiv & \int_{\Omega} \psi(\xi) G(x, t ; \xi, 0) d \xi \\
& +\int_{0}^{t} \int_{\Omega}\left[f_{1}(v(\xi, \tau)) \chi_{D}+f_{2}(v(\xi, \tau)) \chi_{D^{c}}\right] G(x, t ; \xi, \tau) d \xi d \tau
\end{aligned}
$$


Since $f_{1}(\cdot)$ and $f_{2}(\cdot)$ are Lipschitz continuous, one can directly verify that $\mathscr{K}$ is a contraction mapping under the norm $\|v \mid\| \equiv \sup _{0 \leq t \leq T}\left(\|v(\cdot, t)\|_{C^{0}(\Omega)} e^{-A t}\right)$ when $A$ is large enough. Therefore $\mathscr{K}$ has a unique fixed point $v$, which is also the unique solution of (5.1)-(5.3).

Now we turn to establish (5.5). Write $v$ as (5.7)

$$
\begin{aligned}
v(x, t)= & \int_{\Omega} \psi(\xi) G(x, t ; \xi, 0) d \xi+\int_{0}^{t} \int_{\Omega} f_{2}(v(\xi, \tau)) G(x, t ; \xi, \tau) d \xi d \tau \\
& +\int_{0}^{t} \int_{D^{\tau}}\left[f_{1}(v(\xi, \tau))-f_{2}(v(\xi, \tau))\right] H(x, t ; \xi, \tau) d \xi d \tau \\
& +\int_{0}^{t} \int_{D^{\tau}}\left[f_{1}(v(\xi, \tau))-f_{2}(v(\xi, \tau))\right] \Gamma(x-\xi, t-\tau) d \xi d \tau \\
\equiv & w_{1}(x, t)+w_{2}(x, t)+w_{3}(x, t)+w_{4}(x, t) .
\end{aligned}
$$

The function $w_{1}(x, t) \equiv \int_{\Omega} \psi(\xi) G(x, t ; \xi, 0) d \xi$ is in $W_{\infty}^{2,1}\left(\bar{\Omega}_{T}\right)$ since $\psi \in$ $C^{2}(\bar{\Omega})$ satisfies the compatibility condition $\partial_{n} \psi=0$ on $\partial \Omega$. Since the $L^{p}$ estimate (5.4) implies that $v$ is Hölder continuous, the Schauder estimate then implies that $w_{2}(x, t) \equiv \int_{0}^{t} \int_{\Omega} f_{2}(v(\xi, \tau)) G(x, t ; \xi, \tau) d \xi d \tau$ is in $C^{2+\alpha, 1+\alpha / 2}\left(\bar{\Omega}_{T}\right)$. Note that the smoothness of the boundary $\partial \Omega$ and the compactness of the set $\bar{D}$ in $\Omega_{T}$ imply that the function $H(x, t ; \xi, \tau)$ is smooth in the set $\left(\bar{\Omega}_{T}\right) \times \bar{D}$; it follows that the function

$$
w_{3}(x, t) \equiv \int_{0}^{t} \int_{D^{\tau}}\left[f_{1}(v(\xi, \tau))-f_{2}(v(\xi, \tau))\right] H(x, t ; \xi, \tau) d \xi d \tau
$$

is in $C^{2,1}\left(\bar{\Omega}_{T}\right)$. It now remains to show that the function

$$
w_{4}(x, t) \equiv \int_{0}^{t} \int_{D^{\tau}} g(\xi, \tau) \Gamma(x-\xi, t-\tau) d \xi d \tau
$$

is in $W_{\infty}^{2,1}\left(\bar{\Omega}_{T}\right)$, where $g(\xi, \tau) \equiv f_{1}(v(\xi, \tau))-f_{2}(v(\xi, \tau))$ is Hölder continuous.

If $x \notin \Gamma_{t}$, one can compute

$$
\begin{aligned}
\frac{\partial w_{4}}{\partial x_{i} \partial x_{j}}(x, t)= & \int_{0}^{t} \int_{D \tau}(g(\xi, \tau)-g(x, t)) \Gamma_{x_{i} x_{j}}(x-\xi, t-\tau) d \xi d \tau \\
& +g(x, t) \int_{0}^{t} \int_{\Gamma^{\tau}} n_{i}(\xi, \tau) \Gamma_{\xi_{j}}(x-\xi, t-\tau) d S_{\xi} d \tau \\
\equiv & I_{i j}+g(x, t) J_{i j} \quad \forall 1 \leq i \leq j \leq n,
\end{aligned}
$$

where $d S_{\xi}$ is the surface element of $\Gamma^{\tau}$ and $n_{i}(\xi, \tau)$ is the $i$ th component of $\vec{n}(\xi, \tau)$, the unit outward normal to $\partial D^{\tau}\left(=\Gamma^{\tau}\right)$ at $\xi$. Since $g$ is Hölder continuous, the improper integral $I_{i j}$ is uniformly convergent and uniformly bounded.

To estimate $J_{i j}$, we need only consider the case when $x$ is close to $\Gamma^{t}$. Let $\delta$ be a small constant and assume that $x$ is in a $\delta$-neighborhood of $\Gamma^{t}$. Let $\xi_{0}$ be a point on $\Gamma^{t}$ such that $\left|x-\xi_{0}\right|=\operatorname{dist}\left(x, \Gamma^{t}\right)$. Without loss of generality, we can assume that $\xi_{0}$ is the origin and $\vec{n}\left(\xi_{0}, t\right)=-\vec{e}_{n}$. Note that $x-\xi_{0}$ is parallel to $\vec{n}\left(\xi_{0}, t\right)$, so that $x=\left(0^{\prime}, a\right)$, where $0^{\prime}$ is the origin of $\mathscr{R}^{n-1}$ and 
$|a|<\delta$. Since $\Gamma$ is $C^{1+\alpha,(1+\alpha) / 2}$, there exists a $C^{1+\alpha,(1+\alpha) / 2}$ function $k$ such that in the neighborhood $Q(\delta, t)$, where

$$
Q(\delta, t) \equiv\left\{\left(\xi^{\prime}, \xi_{n}, \tau\right)|| \xi^{\prime}|\leq \delta,| \xi_{n} \mid \leq \delta, \max \left\{0, t-\delta^{2}\right\} \leq \tau \leq t\right\}
$$

the hypersurface $\Gamma^{\tau}$ can be represented by

$\xi_{n}=k\left(\xi^{\prime}, \tau\right), \quad\left(\xi^{\prime}, \tau\right) \in Q^{\prime}(\delta, t) \equiv\left\{\left(\xi^{\prime}, \tau\right)|| \xi^{\prime} \mid \leq \delta, \max \left\{0, t-\delta^{2}\right\} \leq \tau \leq t\right\}$.

Clearly, $k\left(0^{\prime}, t\right)=0$ and $\nabla_{\xi^{\prime}} k\left(0^{\prime}, t\right)=0^{\prime}$; it follows that there exists a constant $C$ such that

$$
\begin{array}{cl}
\left|\nabla_{\xi^{\prime}} k\left(\xi^{\prime}, \tau\right)\right| \leq C\left(\left|\xi^{\prime}\right|+|t-\tau|^{1 / 2}\right)^{\alpha} & \forall(\xi, \tau) \in Q^{\prime}(\delta, t), \\
\left|k\left(\xi^{\prime}, \tau\right)\right| \leq C\left(\left|\xi^{\prime}\right|+|t-\tau|^{1 / 2}\right)^{1+\alpha} & \forall(\xi, \tau) \in Q^{\prime}(\delta, t)
\end{array}
$$

for some positive constant $C$ depending only on the $C^{1+\alpha,(1+\alpha) / 2}$ norm of $\Gamma$. Write $J_{i j}$ as

$$
J_{i j}=\left\{\iint_{\Gamma \cap Q(\delta, t)}+\iint_{\Gamma \backslash Q(\delta, t)}\right\} n_{i}(\xi, \tau) \Gamma_{\xi_{j}}(x-\xi, t-\tau) d S_{\xi} d \tau \equiv J_{i j}^{1}+J_{i j}^{2}
$$

Clearly, $J_{i j}^{2}$ is bounded since its integrand is uniformly bounded. To estimate $J_{i j}^{1}$, we use the identity $\vec{n}(\xi, \tau) d S_{\xi}=\left(\nabla_{\xi^{\prime}} k\left(\xi^{\prime}, \tau\right),-1\right) d \xi^{\prime}$, obtaining

$$
J_{i j}^{1}=\iint_{Q^{\prime}(\delta, t)} k_{\xi_{i}}\left(\xi^{\prime}, \tau\right) \Gamma_{\xi_{j}}\left(-\xi^{\prime}, a-k\left(\xi^{\prime}, \tau\right), t-\tau\right) d \xi^{\prime} d \tau \quad \text { if } i \neq n
$$

and

$$
J_{n n}^{1}=-\iint_{Q^{\prime}(\delta, t)} \Gamma_{\xi_{n}}\left(-\xi^{\prime}, a-k\left(\xi^{\prime}, \tau\right), t-\tau\right) d \xi^{\prime} d \tau
$$

When $i \neq n, J_{i j}^{1}$ is uniformly bounded since, by (5.8) and (5.6), its integrand is bounded by

$$
\left|k_{\xi_{i}}\left(\xi^{\prime}, \tau\right) \Gamma_{\xi_{j}}\left(\xi^{\prime}, a-k, t-\tau\right)\right| \leq C\left(\left|\xi^{\prime}\right|+|t-\tau|^{1 / 2}\right)^{\alpha} \frac{\left|\xi^{\prime}\right|}{t-\tau} \frac{1}{(t-\tau)^{n / 2}} e^{-\left|\xi^{\prime}\right|^{2} / 4(t-\tau)}
$$

which is uniformly integrable.

To estimate $J_{n n}^{1}$, notice that if $\delta$ is small enough, we have

$$
\begin{aligned}
\left|a-k\left(\xi^{\prime}, \tau\right)\right|^{2} & \geq a^{2} / 2-k^{2} \\
& \geq a^{2} / 2-C\left(\left|\xi^{\prime}\right|+(t-\tau)^{1 / 2}\right)^{2(1+\alpha)} \quad(\text { by }(5.9)) \\
& \geq a^{2} / 2-\left|\xi^{\prime}\right|^{2} / 2-C(t-\tau)^{1+\alpha} ;
\end{aligned}
$$

it follows that

$$
\Gamma\left(\xi^{\prime}, a-k, t-\tau\right) \leq C \frac{1}{(t-\tau)^{n / 2}} e^{-\left(\left|\xi^{\prime}\right|^{2}+a^{2}\right) / 8(t-\tau)},
$$


and

$$
\begin{aligned}
\left|J_{n n}^{1}\right|= & \left|\iint_{Q^{\prime}(\delta, t)} \frac{a-k}{2(t-\tau)} \Gamma\left(-\xi^{\prime}, a-k, t-\tau\right) d \xi^{\prime} d \tau\right| \\
\leq & \iint_{Q^{\prime}(\delta, t)} \frac{|a|}{2(t-\tau)} \Gamma d \xi^{\prime} d \tau+\iint_{Q^{\prime}(\delta, t)} \frac{|k|}{2(t-\tau)} \Gamma d \xi^{\prime} d \tau \\
\leq & C \int_{0}^{t} \frac{|a|}{(t-\tau)^{3 / 2}} e^{-a^{2} / 8(t-\tau)} d \tau \int_{\mathscr{R}^{n-1}} \frac{1}{(t-\tau)^{(n-1) / 2}} e^{-\left|\xi^{\prime}\right|^{2} / 8(t-\tau)} d \xi^{\prime} \\
& +C \iint_{Q^{\prime}(\delta, t)} \frac{\left(\left|\xi^{\prime}\right|+|t-\tau|^{1 / 2}\right)^{1+\alpha}}{(t-\tau)^{n / 2+1}} e^{-\left|\xi^{\prime}\right|^{2} / 8(t-\tau)} d \xi^{\prime} d \tau \leq C .
\end{aligned}
$$

In summary, $D_{x}^{2} w_{4}$ is uniformly bounded (except on $\Gamma$ which is of measure zero), and thus so is $D_{x}^{2} v$. Using equation (5.1), we conclude that $v_{t}$ is also bounded, thereby completing the proof of the theorem.

In the sequel, we shall denote by $\mathscr{H}$ the mapping which maps $l$ into $v$, the solution of $(5.1)-(5.3)$.

Theorem 5.2. Assume that $l^{1}, l^{2} \in C^{1+\alpha,(1+\alpha) / 2}\left(\Gamma^{0} \times[0, T]\right)$, and let $v^{1}=\mathscr{H} l^{1}$ and $v^{2}=\mathscr{H} l^{2}$. Then there exists a constant $c$ which depends only on $\Omega, \Gamma^{0}$, and $M \equiv\left\|l^{1}\right\|_{C^{1+\alpha,(1+\alpha) / 2}\left(\Gamma^{0} \times[0, T]\right)}+\left\|l^{2}\right\|_{W_{\infty}^{1,0}\left(\Gamma^{0} \times[0, T]\right)}$ such that $v^{1}$ and $v^{2}$ satisfy

$$
\left\|v^{1}-v^{2}\right\|_{W_{\infty}^{1,0}\left(\bar{\Omega}_{T}\right)} \leq C\left\|l^{1}-l^{2}\right\|_{C^{\alpha, \alpha / 2}\left(\Gamma^{0} \times[0, T]\right)} .
$$

Proof. Subtracting the representation of $v_{2}$ in (5.7) from the one for $v_{1}$ yields

$$
\begin{aligned}
v^{1}(x, & t)-v^{2}(x, t) \\
= & \int_{0}^{t} \int_{\Omega}\left\{\left[f_{1}\left(v^{1}\right)-f_{1}\left(v^{2}\right)\right] \chi_{D^{1}}\right. \\
& \left.+\left[f_{2}\left(v^{1}\right)-f_{2}\left(v^{2}\right)\right] \chi_{\left(D^{1}\right)}\right\}\left.\right|_{v^{2}=v^{2}(\xi, \tau)} ^{v^{1}=v^{1}(\xi, \tau)} G(x, t ; \xi, \tau) d \xi d \tau \\
& +\int_{0}^{1}\left\{\int_{D^{\tau^{1}} \backslash D^{\tau^{2}}}-\int_{D^{\tau^{2}} \backslash D^{\tau^{1}}}\right\} \\
& \times\left[f_{1}\left(v^{2}(\xi, \tau)\right)-f_{2}\left(v^{2}(\xi, \tau)\right)\right] G(x, t ; \xi, \tau) d \xi d \tau \\
\equiv & M(x, t)+N(x, t) .
\end{aligned}
$$

Since $f_{1}(\cdot)$ and $f_{2}(\cdot)$ are Lipschitz continuous, it follows that

$$
\begin{aligned}
\|M\|_{W_{\infty}^{1,0}\left(\bar{\Omega}_{t}\right)} \leq & C \int_{0}^{t} \sup _{\xi \in \Omega}\left\{\left|v^{1}(\xi, \tau)-v^{2}(\xi, \tau)\right|\right\} \\
& \quad \times \sup _{x \in \Omega} \int_{\mathscr{R} n}\left[\left|\nabla_{x} G(x, t ; \xi, \tau)\right|+|G(x, t ; \xi, \tau)|\right] d \xi d \tau \\
\leq & C \int_{0}^{t} \frac{\left\|v_{1}-v_{2}\right\|_{L^{\infty}\left(\bar{\Omega}_{T}\right)}}{\sqrt{t-\tau}} d \tau
\end{aligned}
$$

Suppose that $N$ satisfies

$$
\|N\|_{W_{\infty}^{1,0}\left(\bar{\Omega}_{t}\right)} \leq C\left\|l^{1}-l^{2}\right\|_{C^{\alpha, \alpha / 2}\left(\Gamma^{0} \times[0, t]\right)} ;
$$


then we can substitute (5.12) and (5.13) into (5.11), obtaining

$$
\left\|v^{1}-v^{2}\right\|_{W_{\infty}^{1,0}\left(\bar{\Omega}_{t}\right)} \leq C \int_{0}^{t} \frac{\left\|v^{1}-v^{2}\right\|_{L^{\infty}\left(\bar{\Omega}_{\tau}\right)}}{\sqrt{t-\tau}} d \tau+C\left\|l^{1}-l^{2}\right\|_{C^{\alpha, \alpha / 2}\left(\Gamma^{0} \times[0, t]\right)} .
$$

Inequality (5.10) thus follows by Gronwall's inequality.

It remains to prove (5.13). Using the change of variable $\xi=x_{0}(\eta, r)$ we get

$$
N(x, t)=\int_{0}^{t} \int_{\Gamma^{0}} \int_{l^{2}(\eta, \tau)}^{l^{1}(\eta, \tau)} g(\eta, r, \tau) G\left(x, t ; x_{0}(\eta, r), \tau\right) d r d \eta d \tau
$$

where

$$
g(\eta, r, \tau) \equiv \operatorname{det}\left(\frac{\partial x_{0}(\eta, r)}{\partial(\eta, r)}\right)\left[f_{1}\left(v_{2}\left(x_{0}(\eta, r), \tau\right)\right)-f_{2}\left(v_{2}\left(x_{0}(\eta, r), \tau\right)\right)\right]
$$

Denote by $m=m(t)$ the norm $\left\|l^{1}-l^{2}\right\|_{L^{\infty}\left(\Gamma^{0} \times[0, t]\right)}$. We can estimate $N$ by

$$
\begin{aligned}
|N(x, t)| & \leq m\|g\|_{C^{0}\left(\Gamma^{0} \times[-L, L] \times[0, t]\right)} \int_{0}^{t} \int_{\Gamma^{0}} \sup _{r \in[-L, L]} G\left(x, t ; x_{0}(\eta, r), \tau\right) d \eta d \tau \\
& \leq C m \sqrt{t} .
\end{aligned}
$$

Therefore the $L^{\infty}$ norm of $N$ in $\Omega \times(0, t)$ is bounded by $C m \sqrt{t}$.

For any integer $i, 1 \leq i \leq n$, we can compute

$$
\begin{aligned}
N_{x_{i}}(x, t)= & \int_{0}^{t} \int_{\Gamma^{0}} \int_{l^{2}(\eta, \tau)}^{l^{1}(\eta, \tau)} g(\eta, r, \tau) G_{x_{i}}\left(x, t ; x_{0}(\eta, r), \tau\right) d r d \eta d \tau \\
= & \int_{0}^{t} \int_{\Gamma^{0}} \int_{l^{2}(\eta, \tau)}^{l^{1}(\eta, \tau)}[g(\eta, r, \tau)-g(x, t)] G_{x_{i}}\left(x, t ; x_{0}(\eta, r), \tau\right) d r d \eta d \tau \\
& +g(x, t) \int_{0}^{t} \int_{\Gamma^{0}} \int_{l^{2}(\eta, \tau)}^{l^{1}(\eta, \tau)} H_{x_{i}}\left(x, t ; x_{0}(\eta, r), \tau\right) d r d \eta d \tau \\
& +g(x, t) \int_{0}^{t} \int_{\Gamma^{0}} \int_{l^{2}(\eta, \tau)}^{l^{1}(\eta, \tau)} \Gamma_{x_{i}}\left(x-x_{0}(\eta, r), t-\tau\right) d r d \eta d \tau \\
\equiv & N_{i}^{1}(x, t)+g(x, t) N_{i}^{2}(x, t)+g(x, t) N_{i}^{3}(x, t) .
\end{aligned}
$$

Similar to the $L^{\infty}$ estimate of $N$, one can estimate the $L^{\infty}$ norms of $N_{i}^{1}$ and $N_{i}^{2}$ by $\mathrm{Cm}$. It therefore remains to estimate $N_{i}^{3}$. After applying the same argument as in the case of $J_{i j}$, w need only consider the integral

$$
\bar{N}_{i} \equiv \iint_{Q^{\prime}(\delta, t)} \int_{l_{1}(\eta, \tau)}^{l^{2}(\eta, \tau)} \Gamma_{x_{i}}(\eta, r-a, t-\tau) d r d \eta d \tau
$$

where $|a|<\delta$ and

$$
l^{1}\left(0^{\prime}, t\right)=0, \quad \nabla_{\eta} l^{1}\left(0^{\prime}, t\right)=0^{\prime}
$$


We can estimate $\bar{N}_{n}$ by

$$
\begin{aligned}
\left|\bar{N}_{n}\right|= & \left|\iint_{Q^{\prime}(\delta, t)} \int_{0}^{l^{2}-l^{1}} \frac{\left(a-r-l^{1}\right)}{2(t-\tau)} \Gamma\left(\eta, r+l^{1}-a, t-\tau\right) d r d \eta d \tau\right| \\
\leq & \int_{-m}^{m} d r \iint_{Q^{\prime}(\delta, t)} \frac{|a-r|}{2(t-\tau)} \Gamma\left(\eta, r+l^{1}-a, t-\tau\right) d \eta d \tau \\
& +\int_{-m}^{m} d r \iint_{Q^{\prime}(\delta, t)} \frac{\left|l^{1}(\eta, \tau)\right|}{2(t-\tau)} \Gamma\left(\eta, r+l^{1}-a, t-\tau\right) d \eta d \tau \\
\leq & C m
\end{aligned}
$$

since $\left|l^{1}\right| \leq\left(|\eta|+\sqrt{t-\tau}^{1+\alpha} \sqrt{t-2}\right) \quad($ by $(5.14))$ and

$$
\Gamma\left(\eta, r+l^{1}-a, t-\tau\right) \leq C \frac{1}{(t-\tau)^{n / 2}} e^{-\left(\eta^{2}+|r-a|^{2}\right) / 8(t-\tau)} .
$$

To estimate $\bar{N}_{i}, i<N$, we write $\bar{N}_{i}$ as

$$
\begin{aligned}
\bar{N}_{i}= & \iint_{Q^{\prime}(\delta, t)} \int_{l^{1}(\eta, \tau)}^{l^{2}(\eta, \tau)} \Gamma_{\eta_{i}}(\eta, r-a, t-\tau) d r d \eta d \tau \\
= & \iint_{Q^{\prime}(\delta, t)} \frac{d}{d \eta_{i}}\left\{\int_{l^{1}}^{l^{2}}\left[\Gamma(\eta, r-a, t-\tau)-\Gamma\left(\eta, l^{1}-a, t-\tau\right)\right] d r\right\} d \eta d \tau \\
& -\iint_{Q^{\prime}(\delta, t)} \int_{l^{1}}^{l^{2}} \Gamma_{r}(\eta, r-a, t-\tau) l_{\eta^{\prime}}^{2} d r d \eta d \tau \\
& +\iint_{Q^{\prime}(\delta, t)}\left(l^{2}-l^{1}\right) \frac{d}{d \eta_{i}}\left(\Gamma\left(\eta, l^{1}-a, t-\tau\right)\right) d \eta d \tau .
\end{aligned}
$$

By the divergence theorem, the first integral on the right-hand side of the second equality is bounded. The second integral is also bounded by the same treatment as for the $L^{\infty}$ estimate of $\bar{N}_{n}$. The last integral can be written as

$$
\begin{aligned}
& \iint_{Q^{\prime}\left(0^{\prime}, t\right)}\left(l^{2}\left(0^{\prime}, t\right)-l^{1}\left(0^{\prime}, t\right)\right) \frac{d}{d \eta^{i}}\left(\Gamma\left(\eta, l^{1}-a, t-\tau\right)\right) d \eta d \tau \\
& \quad+\iint_{Q^{\prime}(\delta, t)}\left[\left(l^{2}-l^{1}\right)(\eta, \tau)-\left(l^{2}-l^{1}\right)\left(0^{\prime}, t\right)\right] \frac{d}{d \eta_{i}}\left(\Gamma\left(\eta, l^{1}-a, t-\tau\right)\right) d \eta d \tau .
\end{aligned}
$$

Again, by the divergence theorem, the first integral is bounded by $\mathrm{Cm}$. Since

$$
\left|\left(l^{2}-l^{1}\right)(\eta, t)-\left(l^{2}-l^{1}\right)\left(0^{\prime}, t\right)\right| \leq\left\|l^{1}-l^{2}\right\|_{C^{\alpha, \alpha / 2}}\left(|\eta|+(t-\tau)^{1 / 2}\right)^{\alpha},
$$

the second integral is bounded by $C\left\|l^{1}-l^{2}\right\|_{C^{\alpha, \alpha / 2}}$, and therefore so is $\bar{N}_{i}$. This proves (5.13) and completes the proof of the theorem.

Proof of Theorem 2. Set $\mathscr{F} \equiv \mathscr{G} \circ \mathscr{H}$ and

$$
X_{T_{0}} \equiv\left\{l \in C^{3 / 2}\left(\Gamma^{0} \times\left[0, T_{0}\right]\right) \mid\|l\|_{C^{3 / 2}\left(\Gamma^{0} \times\left[0, T_{0}\right]\right)} \leq L_{0} / 2\right\},
$$

where $L_{0}$ is the constant introduced in $\S 4$ and $T_{0}$ is a small positive constant to be determined. 
By Theorem 5.1, for every $l \in X_{T_{0}},|\mathscr{H}|_{W_{\infty}^{2,1}\left(\bar{\Omega} \times\left[0, T_{0}\right]\right)} \leq M$ for some constant $M$ independent of $l$ and $T_{0}$ (if $T_{0}$ is small). Thus, by Theorem 4.4, $\mathscr{F} l=\mathscr{G}(\mathscr{H} l) \in W_{\infty}^{2,2}\left(\Gamma^{0} \times\left[0, \min \left\{T_{0}, T(M)\right\}\right]\right)$ and

$$
\|\mathscr{F} l\|_{C^{3 / 2}\left(\Gamma^{0} \times\left[0, \min \left\{T_{0}, T(M)\right\}\right]\right)} \leq K(M) T_{0}^{1 / 4}
$$

Hence, if $T_{0}$ is small enough, $\mathscr{F}$ maps $X_{T_{0}}$ into itself. In addition, $\mathscr{F}$ is compact since it maps $X_{T_{0}}$ into a bounded set in $W_{\infty}^{2,2}\left(\Gamma^{0} \times\left[0, T_{0}\right]\right)$ which is compact in $X_{T_{0}}$. Furthermore, by Theorem 4.5, Theorem 5.2, and the interpolation formula (4.35), $\mathscr{F}$ is also continuous. We can therefore apply the Schauder fixed point theorem to conclude that $\mathscr{F}$ has a fixed point which is clearly a solution of the limit free boundary problem.

To prove the uniqueness, assume that $\mathscr{F}$ has two fixed points $l^{1}$ and $l^{2}$. Then it follows from Theorem 4.5 and Theorem 5.2 that

$$
\begin{aligned}
\left\|l^{1}-l^{2}\right\|_{C^{1}\left(\Gamma^{0} \times[0, t]\right)} & \leq C t\left\|\mathscr{H} l^{1}-\mathscr{H} l^{2}\right\|_{W_{\infty}^{1,0}\left(\bar{\Omega}_{t}\right)} \\
& \leq C t\left\|l^{1}-l^{2}\right\|_{C^{\alpha, \alpha / 2}\left(\Gamma^{0} \times[0, t]\right)} \quad \forall t \in\left[0, T_{0}\right] .
\end{aligned}
$$

Therefore $l^{1}=l^{2}$ when $t$ is small. By repeating the above procedure step by step, we can prove the uniqueness of the solution of the limit free boundary problem up to time $T_{0}$; this finishes the proof of Theorem 2.

Remark 5.1. One can see that the estimates in Theorem 4.5 and Theorem 5.2 are stronger than what is needed by the proof of Theorem 2 . These estimates can be used to show the uniqueness of other free boundary problems such as the free boundary problem (1.7) (with $\varepsilon>0$ ).

Remark 5.2. The assertion of Theorem 2 is local (in time) since Theorem 4.4 establishes only the local existence of the classical solution of the motion problem. Although global weak solutions of the motion problem can be obtained by using a so-called "vanishing viscosity" method developed by Crandall, Evans, and Lions [13] and in fact this problem has been well studied by Sethian [32], Osher-Sethian [29], and Barles [4] (also see the references therein), we still cannot get the unique global (weak) solution of the limit free boundary problem since, as we can see, the other arguments for establishing the existence for the solution of the limit free boundary problem do not follow. Very recently, Giga, Goto, and Ishii [25] established the global existence of at least a weak solution to the free boundary problem.

\section{A COMPARISON LEMMA FOR PARABOLIC SYSTEMS AND PROOF OF THEOREM 3}

Lemma 6.1 (Comparison). Let $\left(u^{\varepsilon}, v^{\varepsilon}\right)$ be a solution of the system (2.1)-(2.6). Assume that $f, g \in C^{1}\left(\mathscr{R}^{2}\right)$ satisfy

$$
\frac{\partial f}{\partial v}(u, v) \leq 0, \quad \frac{\partial g}{\partial u}(u, v) \geq 0 \quad \forall(u, v) \in \mathscr{R}^{2}
$$


and that a four-tuple $(\bar{u}, \bar{v}, \underline{u}, \underline{v})$ satisfies

$$
\begin{aligned}
\bar{u}_{t}-\varepsilon \Delta \bar{u}-\frac{1}{\varepsilon} f(\bar{u}, \underline{v}) \geq 0 & \text { in } \Omega_{T}, \\
\bar{v}_{t}-\Delta \bar{v}-g(\bar{u}, \bar{v}) \geq 0 & \text { in } \Omega_{T}, \\
\underline{u}_{t}-\varepsilon \Delta \underline{u}-\frac{1}{\varepsilon} f(\underline{u}, \bar{v}) \leq 0 & \text { in } \Omega_{T}, \\
\underline{v}_{t}-\Delta \underline{v}-g(\underline{u}, \underline{v}) \leq 0 & \text { in } \Omega_{T}, \\
\partial_{n} \underline{u} \leq 0 \leq \partial_{n} \bar{u} & \text { on } \partial \Omega_{T}, \\
\partial_{n} \underline{v} \leq 0 \leq \partial_{n} \bar{v} & \text { on } \partial \Omega_{T}, \\
\underline{u}(x, 0) \leq u^{\varepsilon}\left(x, t_{0}\right) \leq \bar{u}(x, 0) & \text { on } \Omega \times\{0\}, \\
\underline{v}(x, 0) \leq v^{\varepsilon}\left(x, t_{0}\right) \leq \bar{v}(x, 0) & \text { on } \Omega \times\{0\}
\end{aligned}
$$

for some $t_{0} \geq 0$. Then

$$
\begin{array}{ll}
\underline{u}(x, t) \leq u^{\varepsilon}\left(x, t+t_{0}\right) \leq \bar{u}(x, t) & \forall(x, t) \in \Omega_{T}, \\
\underline{v}(x, t) \leq v^{\varepsilon}\left(x, t+t_{0}\right) \leq \bar{v}(x, t) & \forall(x, t) \in \Omega_{T} .
\end{array}
$$

Proof. The assertion of the lemma follows from standard routine techniques, namely, subtracting the differential inequalities (6.2)-(6.5) from their corresponding differential equations satisfied by $u^{\varepsilon}$ and $v^{\varepsilon}$, multiplying the resulting inequalities by $\left(u^{\varepsilon}-\bar{u}\right)^{+}\left(\equiv \max \left\{\left(u^{\varepsilon}-\bar{u}\right), 0\right\}\right),\left(\underline{u}-u^{\varepsilon}\right)^{+},\left(v^{\varepsilon}-\bar{v}\right)^{+}$, and $\left(\underline{v}-v^{\varepsilon}\right)^{+}$respectively, integrating over $\Omega \times(0, t)$ and adding the resulting inequalities together, then using (6.1) and Cauchy's inequality, and finally using the Gronwall inequality to deduce that $\left(u^{\varepsilon}-\bar{u}\right)^{+}=\left(\underline{u}-u^{\varepsilon}\right)^{+}=\left(v^{\varepsilon}-\bar{v}\right)^{+}=$ $\left(\underline{v}-v^{\varepsilon}\right)^{+}=0$.

Remark 6.1. Similar (and simpler) comparison lemmas can be obtained for the other cases where $f$ is monotone in $v$ and $g$ is monotone in $u$.

We call any four-tuple $(\bar{u}, \bar{v}, \underline{u}, \underline{v})$ satisfying (6.2)-(6.9) a sup-subsolution for the system (2.1)-(2.6).

Proof of Theorem 3. Let $(\Gamma, u, v)$ be the solution of the limit free boundary problem in the interval $\left[0, T_{0}\right]\left(T_{0}\right.$ is as in Theorem 2$)$. As before, we denote by $D^{t}$ the interior of $\Gamma^{t}$ and by $D$ the union $\bigcup_{0 \leq t \leq T_{0}}\left(D^{t} \times\{t\}\right)$. Let $d(x, t) \in W_{\infty}^{2,2}\left(\Omega_{T_{0}}\right)$ be a smoothly truncated approximation of the signed distance function from $x$ to $\Gamma^{t}$; more precisely,

$$
d(x, t)= \begin{cases}\frac{3}{4} L_{1} & \text { if } x \in D^{t} \text { and } \operatorname{dist}\left(x, \Gamma^{t}\right) \geq L_{1}, \\ \operatorname{dist}\left(x, \Gamma^{t}\right) & \text { if } x \in D^{t} \text { and } \operatorname{dist}\left(x, \Gamma^{t}\right) \leq \frac{1}{2} L_{1}, \\ -\operatorname{dist}\left(x, \Gamma^{t}\right) & \text { if } x \in \bar{\Omega} \backslash D^{t} \text { and } \operatorname{dist}\left(x, \Gamma^{t}\right) \leq \frac{1}{2} L_{1}, \\ -\frac{3}{4} L_{1} & \text { if } x \in \bar{\Omega} \backslash D^{t} \text { and } \operatorname{dist}\left(x, \Gamma^{t}\right) \geq L_{1},\end{cases}
$$

where $L_{1} \in\left(0, L_{0}\right)$ is a fixed small constant. We can assume that $|d(x, t)| \geq$ $L_{1} / 2$ whenever $\operatorname{dist}\left(x, \Gamma^{t}\right)>L_{1} / 2$. Taking smaller $L_{1}$ if necessary, we may also assume that $\operatorname{dist}\left(\partial \Omega, \Gamma^{t}\right)>L_{1}$ for all $t \in\left[0, T_{0}\right]$. It follows that

$$
\partial_{n} d(x, t)=0 \quad \forall(x, t) \in \partial \Omega_{T_{0}} .
$$

Let $(U(\cdot, v), \mathscr{V}(v))$ be the unique solution of the eigenvalue problem $(1.5)$. 
Direct verification shows that when $f$ is given by (1.3), the solution is given by

$$
\begin{aligned}
U(z, v) & =h_{+}(v)-\frac{h_{+}(v)-h_{-}(v)}{1+\exp \left[\frac{1}{\sqrt{2}}\left(z+z_{0}(v)\right)\left(h_{+}(v)-h_{-}(v)\right)\right]}, \\
\mathscr{V}(v) & =\frac{1}{\sqrt{2}}\left(2 h_{0}(v)-h_{+}(v)-h_{-}(v)\right),
\end{aligned}
$$

where $z_{0}(v) \in \mathscr{R}^{1}$ is a constant ensuring the last condition in (1.5). Clearly, the function $U$ satisfies

$$
\begin{aligned}
\left|U_{z}(z, v)\right|+\left|U_{z z}(z, v)\right|+\left|U(z, v)-h_{+}(v)\right| \leq A \exp (-\alpha z) & \forall z>0, \\
(6.15)\left|U_{z}(z, v)\right|+\left|U_{z z}(z, v)\right|+\left|U(z, v)-h_{-}(v)\right| \leq A \exp (\alpha z) & \forall z<0
\end{aligned}
$$

for some positive constants $A$ and $\alpha$ which are independent of $v$ if $v$ is in a compact subset of $(-2 \sqrt{3} / 9,2 \sqrt{3} / 9)$.

Remark 6.2. The particular choice of $f$ in (1.3) is only for convenience; the inequalities (6.13)-(6.15) hold also for general double well-potential $f$.

Set $t_{0}=\tau_{0} \varepsilon|\ln \varepsilon|$, where $\tau_{0}$ is as in Theorem 1 , and define

$$
\begin{gathered}
\bar{v}=v+h, \\
\underline{v}=v-h, \\
\bar{u}=U\left(\frac{d+M_{1} \varepsilon|\ln \varepsilon| e^{M t}}{\varepsilon}, v-2 h^{\varepsilon}\right), \\
\underline{u}=U\left(\frac{d-M_{1} \varepsilon|\ln \varepsilon| e^{M t}}{\varepsilon}, v+2 h\right),
\end{gathered}
$$

where $M$ and $M_{1}$ are (large) constants independent of $\varepsilon$, and $h$ is a positive function depending on $\varepsilon$. We shall choose appropriate $M, M_{1}$, and $h$ such that $(\bar{u}, \bar{v}, \underline{u}, \underline{v})$ defined in (6.16)-(6.19) satisfy (6.2)-(6.9), and therefore we can use Lemma 6.1 to conclude that

$$
\begin{array}{ll}
\underline{u}(x, t) \leq u^{\varepsilon}\left(x, t+\tau_{0} \varepsilon|\ln \varepsilon|\right) \leq \bar{u}(x, t) & \forall(x, t) \in \bar{\Omega}_{T_{0}}, \\
\underline{v}(x, t) \leq v^{\varepsilon}\left(x, t+\tau_{0} \varepsilon|\ln \varepsilon|\right) \leq \bar{v}(x, t) & \forall(x, t) \in \bar{\Omega}_{T_{0}} .
\end{array}
$$

First, we verify the boundary conditions (6.6) and (6.7). Assume that

$$
\partial_{n} h(x, t)=0 \quad \forall(x, t) \in \partial \Omega_{T_{0}} .
$$

Then, observing that $\partial_{n} v=\partial_{n} d=0$ on $\partial \Omega_{T_{0}}$, we immediately obtain

$$
\partial_{n} \bar{u}=\partial_{n} \bar{v}=\partial_{n} \underline{u}=\partial_{n} \underline{v}=0 \quad \forall(x, t) \in \partial \Omega_{T_{0}},
$$

i.e., inequalities (6.6) and (6.7) hold if $h$ satisfies (6.22).

Next, we verify the initial conditions (6.8) and (6.9). that

It follows from (2.9) and the definition of $\bar{v}$ and $\underline{v}$ that (6.9) holds provided

$$
h(x, 0) \geq C_{0} \tau_{0} \varepsilon|\ln \varepsilon| .
$$

To establish the second inequality in (6.8), let $c_{0}$ be the constant in (2.20) and consider two cases:

(i) $d(x, t) \leq-\frac{M_{0}}{c_{0}} \varepsilon|\ln \varepsilon|$ and

(ii) $d(x, t)>-\frac{M_{0}}{c_{0}} \varepsilon|\ln \varepsilon|$. 
In the first case, (2.20) implies that $\varphi(x) \leq h_{0}(\psi(x))-M_{0} \varepsilon|\ln \varepsilon|$, i.e., $x \in$ $\Omega_{M_{0} \varepsilon|\ln \varepsilon|}^{+}$. It follows from Theorem 1 that

$$
u^{\varepsilon}\left(x, \tau_{0} \varepsilon|\ln \varepsilon|\right) \leq h_{-}(\psi(x))+M_{0} \varepsilon|\ln \varepsilon| .
$$

Observe that the functions $h_{-}(\cdot)$ and $h_{+}(\cdot)$ are monotone decreasing and there exists a positive constant $c_{1}$ satisfying

$$
h_{ \pm}(b-a) \geq h_{ \pm}(b)+c_{1} a \quad \forall b \in\left[-\frac{2 \sqrt{3}}{9}+\frac{\sigma}{2}, \frac{2 \sqrt{3}}{9}-\frac{\sigma}{2}\right], a \in\left[0, \frac{\sigma}{4}\right]
$$

it follows that if

$$
h(x, 0) \geq \frac{M_{0}}{c_{1}} \varepsilon|\ln \varepsilon| \quad \forall x \in \Omega,
$$

then

$$
\begin{aligned}
\bar{u}(x, 0) & =U\left(\frac{d+M_{1} \varepsilon|\ln \varepsilon|}{\varepsilon}, \psi-2 h\right) \\
& \geq h_{-}(\psi-2 h) \geq h_{-}(\psi)+2 c_{1} h(x, 0) \\
& \geq h_{-}(\psi)+2 M_{0} \varepsilon|\ln \varepsilon| \geq u^{\varepsilon}\left(x, \tau_{0} \varepsilon|\ln \varepsilon|\right),
\end{aligned}
$$

i.e., the second inequality in (6.8) holds.

To get the second inequality in (6.8) in case (ii), let $\alpha$ be the positive constant in (6.14), and set

$$
M_{1} \equiv \frac{M_{0}}{c_{0}}+\frac{2}{\alpha} .
$$

Then in case (ii), we can compute

$$
\begin{aligned}
\bar{u}(x, 0) & =U\left(\frac{d+M_{1} \varepsilon|\ln \varepsilon|}{\varepsilon}, \psi-2 h\right) \\
& \geq U\left(\frac{2}{\alpha}|\ln \varepsilon|, \psi-2 h\right) \geq h_{+}(\psi-2 h)-A e^{-2|\ln \varepsilon|} \\
& \geq h_{+}(\psi)+2 c_{1} h(x, 0)-A \varepsilon^{2} \geq h_{+}(\psi)+2 M_{0} \varepsilon|\ln \varepsilon|-A \varepsilon^{2} \\
& \geq h_{+}(\psi)+M_{0} \varepsilon|\ln \varepsilon| \geq u^{\varepsilon}\left(x, \tau_{0} \varepsilon|\ln \varepsilon|\right),
\end{aligned}
$$

where we have used the monotonicity of $U(\cdot, v)$ in the first inequality, (6.14) in the second inequality, (6.25) in the third inequality, (6.26) in the fourth inequality, and Theorem 1 in the last inequality. Therefore, the second inequality in (6.8) holds. Similarly, we can prove that the first inequality in (6.8) holds under the condition (6.26) and the choice of $M_{1}$ in (6.27).

Finally, we verify the differential inequalities $(6.2)-(6.5)$.

First we consider (6.2). Denote by $\bar{U}, \bar{U}_{z}, \bar{U}_{v}$, etc. the functions $U$, $U_{z}, U_{v}$, etc., evaluated at $\left(\left(d+M_{1} \varepsilon|\ln \varepsilon| e^{M t}\right) / \varepsilon, v-2 h\right)$. Direct computation 
yields

$$
\begin{aligned}
\bar{u}_{t}- & \varepsilon \Delta \bar{u}-\frac{1}{\varepsilon} f(\bar{u}, \underline{v}) \\
= & \left\{\bar{U}_{z} \frac{d_{t}+M M_{1} \varepsilon|\ln \varepsilon| e^{M t}}{\varepsilon}+\bar{U}_{v}\left[v_{t}-2 h_{t}\right]\right\} \\
& -\varepsilon\left\{\bar{U}_{z z} \frac{|\nabla d|^{2}}{\varepsilon^{2}}+\bar{U}_{z} \frac{\Delta d}{\varepsilon}+2 \bar{U}_{z v} \frac{\nabla d \cdot \nabla(v-2 h)}{\varepsilon}+\bar{U}_{v v}|\nabla(v-2 h)|^{2}+\bar{U}_{v} \Delta(v-2 h)\right\} \\
& \left.-\frac{1}{\varepsilon}\{f(\bar{U}, v-2 h)-h\}, \quad+\frac{\bar{U}_{z}}{\varepsilon}\left[d_{t}+M M_{1} \varepsilon|\ln \varepsilon| e^{M t}+\mathscr{V}(u-2 h)\right]\right\}+\left\{\frac{1}{\varepsilon} \bar{U}_{z z}\left(1-|\nabla d|^{2}\right)\right\} \\
& -\left\{\bar{U}_{z} \Delta d+2 \bar{U}_{z v} \nabla d \cdot \nabla(v-2 h)+\varepsilon \bar{U}_{v v}|\nabla(v-2 h)|^{2}\right. \\
\equiv & I_{1}+I_{2}+I_{3}+\frac{h}{\varepsilon} .
\end{aligned}
$$

where in the second equality we have used the equation satisfied by $U$.

To estimate $I_{1}$, notice that

$$
d_{t}(x, t)=-\mathscr{V}(v(x, t)) \quad \forall x \in \Gamma^{t}(=\{x \in \Omega \mid d(x, t)=0\})
$$

since the outward normal velocity of $\Gamma_{t}$ is $d_{t}$ and $(\Gamma, u, v)$ is a solution of the limit free boundary problem. By the mean value theorem and the smoothness of the function $d$, equation (6.28) implies

$$
\left|d_{t}(x, t)+\mathscr{V}(v(x, t))\right| \leq C|d(x, t)| \quad \forall(x, t) \in \bar{\Omega}_{T_{0}} .
$$

It follows that

$$
\begin{aligned}
d_{t}+ & M M_{1} \varepsilon|\ln \varepsilon| e^{M t}+\mathscr{V}(v-2 h) \\
& =d_{t}+\mathscr{V}(v)+M M_{1} \varepsilon|\ln \varepsilon| e^{M t}+[\mathscr{V}(v-2 h)-\mathscr{V}(v)] \\
& \geq-C|d|+M M_{1} \varepsilon|\ln \varepsilon| e^{M t}-C h \\
& \geq-C\left|d+M_{1} \varepsilon\right| \ln \varepsilon\left|e^{M t}\right|+(M-C) M_{1} \varepsilon|\ln \varepsilon| e^{M t}-C h .
\end{aligned}
$$

Therefore if $M$ and $h$ satisfy

$$
\sup _{x \in \Omega}|h(x, t)| \leq(M-C) M_{1} \varepsilon|\ln \varepsilon| e^{M t} \quad \forall t \in\left(0, T_{0}\right],
$$

then

$$
d_{t}+M M_{1} \varepsilon|\ln \varepsilon| e^{M t}+\mathscr{V}(v-2 h) \geq-C\left|d+M_{1} \varepsilon\right| \ln \varepsilon\left|e^{M t}\right| .
$$

It follows that

$$
\begin{aligned}
I_{1} & \geq-C \frac{\left|d+M_{1} \varepsilon\right| \ln \varepsilon\left|e^{M t}\right|}{\varepsilon} U_{z}\left(\frac{d+M_{1} \varepsilon|\ln \varepsilon| e^{M t}}{\varepsilon}, v-2 h\right) \\
& \geq-C \sup _{z \in \mathscr{R}}\left(|z| e^{-\alpha|z|}\right) \geq-C
\end{aligned}
$$

by (6.14) and (6.15). 
Next we estimate $I_{2}$. Since $|\nabla d|=1$ when $|d| \leq L_{1} / 2$, we have

$$
\begin{aligned}
I_{2} & \equiv \frac{1}{\varepsilon} \bar{U}_{z z}\left(1-|\nabla d|^{2}\right) \\
& \geq-\frac{C}{\varepsilon} \sup _{|d| \geq L_{1} / 2}\left|U_{z z}\left(\frac{d+M_{1} \varepsilon|\ln \varepsilon| e^{M t}}{\varepsilon}, v-2 h\right)\right| \\
& \geq-\frac{C}{\varepsilon} e^{-\alpha\left(L_{1} / 2-M_{1} \varepsilon|\ln \varepsilon| e^{M T_{0}}\right) / \varepsilon} \geq-C
\end{aligned}
$$

if $\varepsilon$ is small enough.

Finally, if we assume that $h$ satisfies

$$
\|h\|_{W_{\infty}^{2,1}\left(\Omega_{T_{0}}\right)} \leq C
$$

then $\left|I_{3}\right| \leq C$.

Combining the above estimates for $I_{1}, I_{2}$, and $I_{3}$, we conclude that

$$
\bar{u}_{t}-\varepsilon \Delta \bar{u}-\frac{1}{\varepsilon} f(\bar{u}, \underline{v}) \geq-C+\frac{h}{\varepsilon} .
$$

The right-hand side is nonnegative if $h$ satisfies

$$
h(x, t) \geq C \varepsilon \quad \forall(x, t) \in \Omega_{T_{0}} .
$$

It follows that inequality (6.2) holds if $M$ and $h$ satisfy (6.29), and $h$ satisfies (6.30) and (6.31). Similarly, we can show that inequality (6.4) holds under the same conditions.

Next, we verify the inequality (6.3). Calculation yields

$$
\begin{aligned}
\bar{v}_{t}- & \Delta \bar{v}-g(-\bar{u}, \bar{v}) \\
& =(v+h)_{t}-\Delta(v+h)-g(\bar{u}, v+h) \\
& =g\left(h_{+}(v), v\right) \chi_{D}+g\left(h_{-}(v), v\right) \chi_{\Omega_{T_{0} \backslash D}+h_{t}-\Delta h-g(\bar{u}, v+h)} \\
& =\left(h_{+}(v)-\bar{u}\right) \chi_{\{d>0\}}+\left(h_{-}(v)-\bar{u}\right) \chi_{\{d \leq 0\}}+h_{t}-\Delta h+\gamma h,
\end{aligned}
$$

where we have used (1.4) in the last equality. We can estimate the first term on the right-hand side of the last equality by

$$
\begin{aligned}
\left|h_{+}(v)-\bar{u}\right| \chi_{\{d>0\}} & \leq\left|\bar{u}-h_{+}(v-2 h)\right| \chi_{\{d>0\}}+\left|h_{+}(v-2 h)-h_{+}(v)\right| \\
& \leq A e^{-\alpha M_{1}|\ln \varepsilon|}+C h \leq A \varepsilon^{2}+C h
\end{aligned}
$$

by the definition of $\bar{u}$ and (6.14). Similarly, we can estimate the second term in $(6.32)$ by

$$
\begin{aligned}
\left|h_{-}(v)-\bar{u}\right| \chi_{\{d \leq 0\}} \leq & \left|\bar{u}-h_{-}(v-2 h)\right| \chi_{\left\{d \leq-2 M_{1} \varepsilon|\ln \varepsilon| e^{M t}\right\}} \\
& +C \chi_{\left\{-2 M_{1} \varepsilon|\ln \varepsilon| e^{M t} \leq d \leq 0\right\}}+\left|h_{-}(v-2 h)-h_{-}(v)\right| \\
\leq & A \varepsilon^{2}+C \chi_{\left\{-2 M_{1} \varepsilon|\ln \varepsilon| e^{M t} \leq d \leq 0\right\}}+C h .
\end{aligned}
$$

Substituting these two estimates into (6.32), we find that the differential inequality (6.3) holds if $h$ satisfies the differential inequality 


$$
h_{t}-\Delta h \geq C h+C\left\{\varepsilon+\chi_{\left\{|d| \leq 2 M_{1} \varepsilon|\ln \varepsilon| e^{M t}\right\}}\right\} \quad \forall(x, t) \in \Omega_{T_{0}} .
$$

Similarly, inequality (6.5) holds if $h$ satisfies (6.33).

In summary, $(\bar{u}, \bar{v}, \underline{u}, \underline{v})$ is a sup-subsolution if $M_{1}$ is given by (6.27), $M$ and $h$ satisfy (6.29), and $h$ satisfies (6.22), (6.23), (6.26), (6.30), (6.31), and (6.33).

Let $h$ (depending on $M$ ) be the solution of the following parabolic problem:

(6.35) $\partial_{n} h(x, t)=0$,

$$
(x, t) \in \Omega_{T_{0}},
$$

$$
h(x, 0)=\max \left\{C_{0} \tau_{0}, M_{0} / c_{1}\right\} \varepsilon|\ln \varepsilon|, \quad x \in \Omega .
$$

Clearly, such defined $h$ satisfies the conditions (6.22), (6.23), (6.26), and (6.33). Since

$$
\chi_{\left\{|d| \leq 2 M_{1} \varepsilon|\ln \varepsilon| e^{M t}\right\}}=\chi_{\left\{d \leq 2 M_{1} \varepsilon|\ln \varepsilon| e^{M t}\right\}}-\chi_{\left\{d \leq-2 \varepsilon|\ln \varepsilon| e^{M t}\right\}}
$$

and the boundaries of the sets $\left\{d \leq 2 M_{1} \varepsilon|\ln \varepsilon| e^{M t}\right\}$ and $\left\{d \leq-2 M_{1} \varepsilon|\ln \varepsilon| e^{M t}\right\}$ are smooth (if $\varepsilon$ is small enough), the analysis in $\S 5$ implies that $h \in W_{\infty}^{2,1}\left(\Omega_{T_{0}}\right)$, i.e., $h$ satisfies (6.30). Note that the right-hand side of (6.34) is positive, so that

$$
h(x, t) \geq \min _{\xi \in \Omega}\{h(\xi, 0)\}=\max \left\{C_{0} \tau_{0}, M_{0} / c_{1}\right\} \varepsilon|\ln \varepsilon| \quad \forall(x, t) \in \Omega_{T_{0}},
$$

that is, $h$ satisfies $(6.31)$.

It now remains to verify the condition (6.29). Write $h$ as

$$
\begin{aligned}
& h(x, t)=e^{C t}\left\{\max \left\{C_{0} \tau_{0}, \frac{M_{0}}{c_{1}}\right\} \varepsilon|\ln \varepsilon|+C \varepsilon \int_{0}^{t} \int_{\Omega} G(x, t ; \xi, \tau) d \xi d \tau\right. \\
&+C \int_{0}^{t} \int_{-2 M_{1} \varepsilon|\ln \varepsilon| e^{M_{\tau}}}^{2 M_{1} \varepsilon|\ln \varepsilon| e^{\mathcal{M}_{\tau}}} \int_{\Gamma^{\tau}} G(x, t ; T(\eta, r, \tau), \tau) \\
&\left.\operatorname{det}\left(\frac{\partial T(\eta, r, \tau)}{\partial(\eta, r)}\right) d \eta d r d \tau\right\},
\end{aligned}
$$

where $G$ is the Green function of the heat operator $\partial_{t}-\Delta$ introduced in $\S 5$, $T(\eta, r, \tau)=\eta+r \vec{n}_{\Gamma^{\tau}}(\eta)$ is a diffeomorphism from $\Gamma^{\tau} \times\left(-L_{1} / 2, L_{1} / 2\right)$ to $\left\{x \in \Omega|| d(x, t)<L_{1} / 2\right\}$, and $\vec{n}_{\Gamma^{\tau}}$ is the unit outward normal to $\Gamma^{\tau}$. It follows that

$$
\begin{aligned}
\sup _{x \in \Omega}|h(x, t)| & \leq C e^{C T_{0}}\left\{\varepsilon|\ln \varepsilon|+\varepsilon t+\int_{0}^{t} M_{1} \varepsilon|\ln \varepsilon| e^{M \tau} \frac{1}{(t-\tau)^{1 / 2}} d \tau\right\} \\
& \leq C\left(T_{0}\right)\left\{1+\frac{M_{1}}{M^{1 / 2}} e^{M t}\right\} \varepsilon|\ln \varepsilon| \\
& \leq(M-C) \varepsilon|\ln \varepsilon| e^{M t} \quad \forall t \in\left[0, T_{0}\right]
\end{aligned}
$$

if $M$ is large enough. Inequality (6.29) thus holds.

We have now shown that $\left(\bar{u}, \bar{v}, \underline{u}, \underline{v}\right.$ ) satisfies (6.2)-(6.9) (with $t_{0}=$ $\left.\tau_{0} \varepsilon|\ln \varepsilon|\right)$, and therefore, by Lemma 6.1, (6.20) and (6.21) hold. The assertion of Theorem 3 thus follows from the definition of $(\bar{u}, \bar{v}, \underline{u}, \underline{v}),(6.29),(6.14)$, and (6.15). 


\section{REFERENCES}

1. S. Allen and J. Cahn, A microscopic theory for antiphase boundary motion and its application to antiphase domain coarsing, Acta Metall. 27 (1979), 1084-1095.

2. D. G. Aronson and H. F. Weinberger, Nonlinear diffusion in population genetics, combustion, and nerve propagation, Partial Differential Equations and Related Topics (J. A. Goldstein, ed.), Lecture Notes in Math., vol. 446, Springer-Verlag, New York, 1975, pp. 5-49.

3. Multidimensional nonlinear diffusion arising in population genetics, Adv. Math. 30 (1978), 33-76.

4. G. Barles, Remarks on a flame propagation model, Rapport INRIA, \#464, 1985.

5. L. Bronsard and R. V. Kohn, Motion by mean curvature as the singular limit of GinzburgLandau dynamics, J. Differential Equations 90 (1991), 211-237.

6. _ On the slowness of phase boundary motion in one space dimension, Comm. Pure. Appl. Math. 43 (1990), 983-987.

7. J. Carr and R. Pego, Very slow phase separation in one dimension, (M. Rascle, ed.), Lecture Notes in Phys., vol. 344, Springer-Verlag, 1989, pp. 216-226.

8. _ Invariant manifolds for metastable pattern in $u_{t}=\varepsilon^{2} u_{x x}-f(u)$, Proc. Roy. Soc. Edinburgh 116 (1990), 133-160.

9. Xinfu Chen, Generation and propagation of interface in reaction-diffusion equations, J. Differential Equations 96 (1992), 116-141.

10. Xu-Yan Chen, Dynamics of interfaces in reaction diffusion systems, Hiroshima Math. J. 21 (1991), 47-83.

11. Y. G. Chen, Y. Giga, and S. Goto, Uniqueness and existence of viscosity solutions of generalized mean curvature flow equations, J. Differential Geom. 33 (1991), 749-786.

12. R. Courant and D. Hilbert, Methods of mathematical physics, Vol. II, Wiley, New York, 1962.

13. M. G. Crandall, L. C. Evans, and P. L. Lions, Some properties of viscosity solutions of Hamilton-Jacobi equations, Trans. Amer. Math. Soc. 282 (1984), 487-502.

14. P. DeMottoni and M. Schatzman, Evolution géométrique d'interfaces, C. R. Acad. Sci. Paris Sér. I Math. 309 (1989), 453-458.

15. _ Development of interfaces in $\mathscr{R}^{N}$, preprint.

16. L. C. Evans, H. M. Soner, and P. E. Souganidis, The Allen-Cahn equation and the generalized motion by mean curvature, preprint.

17. L. C. Evans and J. Spruck, Motion of level set by mean curvature. I, J. Differential Geom. 33 (1991), 635-681.

18. P. C. Fife, Dynamics of internal layers and diffusive interfaces, CBMS-NSF Regional Conf. Ser. in Appl. Math., SIAM, Philadelphia, Pa., 1988.

19. P. C. Fife and L. Hsiao, The generation and propagation of internal layers, Nonlinear Anal. 70 (1988), 31-46.

20. P. C. Fife and B. McLeod, The approach of solutions of nonlinear diffusion equation to travelling front solutions, Arch. Rational Mech. Anal. 65 (1977), 335-361.

21. P. C. Fife and J. Tyson, Target patterns in a realistic model of the Belousov-Zhabotinskii reaction, J. Chem. Phys. 73 (1980), 2224-2237.

22. A. Friedman, Partial differential equations of parabolic type, Prentice-Hall, Englewood Cliffs, N.J., 1964.

23. G. Fusco, $A$ geometric approach to the dynamics of $u_{t}=\varepsilon^{2} u_{x x}+f(u)$ for small $\varepsilon(\mathrm{K}$. Kirchgassner, ed.), Lecture Notes in Phys., vol. 359, Springer-Verlag, 1990, pp. 53-73.

24. G. Fusco and J. K. Hale, Slow-motion manifolds, dormant instability, and singular perturbations, J. Dynamics Differential Equations 1 (1989), 75-94.

25. Y. Giga, S. Goto, and H. Ishii, Global existence of weak solutions for interface equations coupled with diffusion equations, IMA Preprint \#806, University of Minnesota, 1991. 
26. D. Hilhorst, Y. Nishiura, and M. Mimura, A free boundary problem arising from reactiondiffusion system, preprint, 1990.

27. O. A. Ladyzhenskaja, V. A. Solonnikov, and N. N. Ural'ceva, Linear and quasilinear equations of parabolic type, Amer. Math. Soc., Providence, R.I., 1968.

28. T. Ohta, M. Mimura, and R. Kobayashi, Higher dimensional localized patterns in excitable media, Physics D 34 (1989), 115-144.

29. S. Osher and J. A. Sethian, Fronts propagating with curvature dependent speed: algorithms based on Hamilton-Jacobi formulations, J. Comput. Phys. 79 (1988), 12-49.

30. J. Rubinstein, P. Sternberg, and J. B. Keller, Fast reaction, slow diffusion and curve shorting, SIAM J. Appl. Math. 49 (1989), 116-133.

31. J. Smoller, Shock waves and reaction-diffusion equations, Springer-Verlag, New York, 1982.

32. J. A. Sethian, Curvature and evolution of fronts, Comm. Math. Phys. 101 (1985), 487-499.

Department of Mathematics, University of Pittsburgh, Pittsburgh, Pennsylvania 15260

E-mail address: xinfu@unix.cis.pitt.edu 